\title{
DISTANCE BETWEEN TWO CIRCLES IN ANY NUMBER OF DIMENSIONS IS A VECTOR ELLIPSE
}

Author: Aslı Pınar Tan ${ }^{\S}$

\begin{abstract}
Based on measured astronomical position data of heavenly objects in the Solar System and other planetary systems, all bodies in space seem to move in some kind of elliptical motion with respect to each other, whereas objects follow parabolic escape orbits while moving away from Earth and bodies asserting a gravitational pull, and some comets move in nearhyperbolic orbits when they approach the Sun. In this article, it is first mathematically proven that the "distance between points on any two different circles in three-dimensional space" is equivalent to the "distance of points on a vector ellipse from another fixed or moving point, as in two-dimensional space." Then, it is further mathematically demonstrated that "distance between points on any two different circles in any number of multiple dimensions" is equivalent to "distance of points on a vector ellipse from another fixed or moving point". Finally, two special cases when the "distance between points on two different circles in multi-dimensional space" become mathematically equivalent to distances in "parabolic" or "near-hyperbolic" trajectories are investigated. Concepts of "vector ellipse", "vector hyperbola", and "vector parabola" are also mathematically defined. The mathematical basis derived in this Article is utilized in the book "Everyhing Is A Circle: A New Model For Orbits Of Bodies In The Universe" in asserting a new Circular Orbital Model for moving bodies in the Universe, leading to further insights in Astrophysics.
\end{abstract}

\section{ARTICLE}

Consider a system of two circles in three-dimensional space, with the geometry of the system demonstrated as in Figure 1 in Cartesian $(\hat{\boldsymbol{x}}, \hat{\boldsymbol{y}}, \hat{\boldsymbol{z}})=\left(\hat{\boldsymbol{u}}_{1}, \hat{\boldsymbol{u}}_{2}, \hat{\boldsymbol{u}}_{3}\right)$ coordinates. The two circles have vector radii $\overrightarrow{\boldsymbol{r}}_{1}(1)-(2)$ and $\overrightarrow{\boldsymbol{r}}_{2}$ (3), with constant magnitudes $r_{1}$ (4) and $r_{2}$ (4), respectively, which are radius vectors at point $\mathbf{P}_{1}$ phased at $\left(\phi+\phi_{0}\right)$ and $\mathbf{P}_{2}$ phased at $(\phi)$ on the two circles, respectively, phased apart by a constant or time $(t)$-dependent angle $\phi_{0}(1)$.

\footnotetext{
$\S$ Asli Pinar Tan

LinkedIn Website: http://www.linkedin.com/in/apinartan

E-mail: aslipinartan@superonline.com
} 
The scalar magnitude $r_{1}(4)$ of the radius vector $\vec{r}_{1}(1)-(2)$ is calculated as the square root of $r_{1}^{2}(4)$, which in turn is calculated in terms of the Dot Product ${ }^{1}\left[\vec{r}_{1} \cdot \vec{r}_{1}\right]$ (4) of the $\vec{r}_{1}$ (1) - (2) vector with itself. In the same way, the scalar magnitude $r_{2}(4)$ of the radius vector $\vec{r}_{2}(3)$ is calculated as the square root of $r_{2}^{2}$ (4), which in turn is calculated in terms of the Dot Product $^{1}\left[\overrightarrow{\boldsymbol{r}}_{2} \cdot \overrightarrow{\boldsymbol{r}}_{2}\right]$ (4) of the vector $\overrightarrow{\boldsymbol{r}}_{2}$ (3) with itself. Centers of these two circles are displaced by a constant or variable vector $\vec{\ell}(\phi)$ (5) with magnitude $\ell(\phi)(6)$ at each phase $\phi$. The scalar distance between the centers of the two circles at every phase $\phi$, namely magnitude $\ell(\phi)$ (6) of $\vec{\ell}(\phi)$ (5), is calculated as the square root of $\ell^{2}(\phi)(6)$, which in turn is calculated in terms of the Dot $\operatorname{Product}^{1}[\vec{\ell}(\phi) \cdot \vec{\ell}(\phi)](6)$ of the vector $\vec{\ell}(\phi)$ (5) with itself.

More explicitly, in the Cartesian $(\hat{\boldsymbol{x}}, \hat{\boldsymbol{y}}, \hat{\boldsymbol{z}})$ coordinate configuration of Figure 1, at each constant or time $(t)$-dependent phase angle $\phi$, the location of $\mathbf{P}_{1}$ phased at constant or time $(t)$-dependent phase angle $\left(\phi+\phi_{0}\right)$ is defined by the vector $\left[\vec{r}_{1}(\phi)+\vec{\ell}(\phi)\right]$ based on (1) - (2) and (5), and the location of $\mathbf{P}_{2}$ phased at $(\phi)$ is defined by the vector $\overrightarrow{\boldsymbol{r}}_{2}(\phi)$ (3). Note that the inclination angle $\beta$ (1) between the planes of these two circles is also taken to be constant.

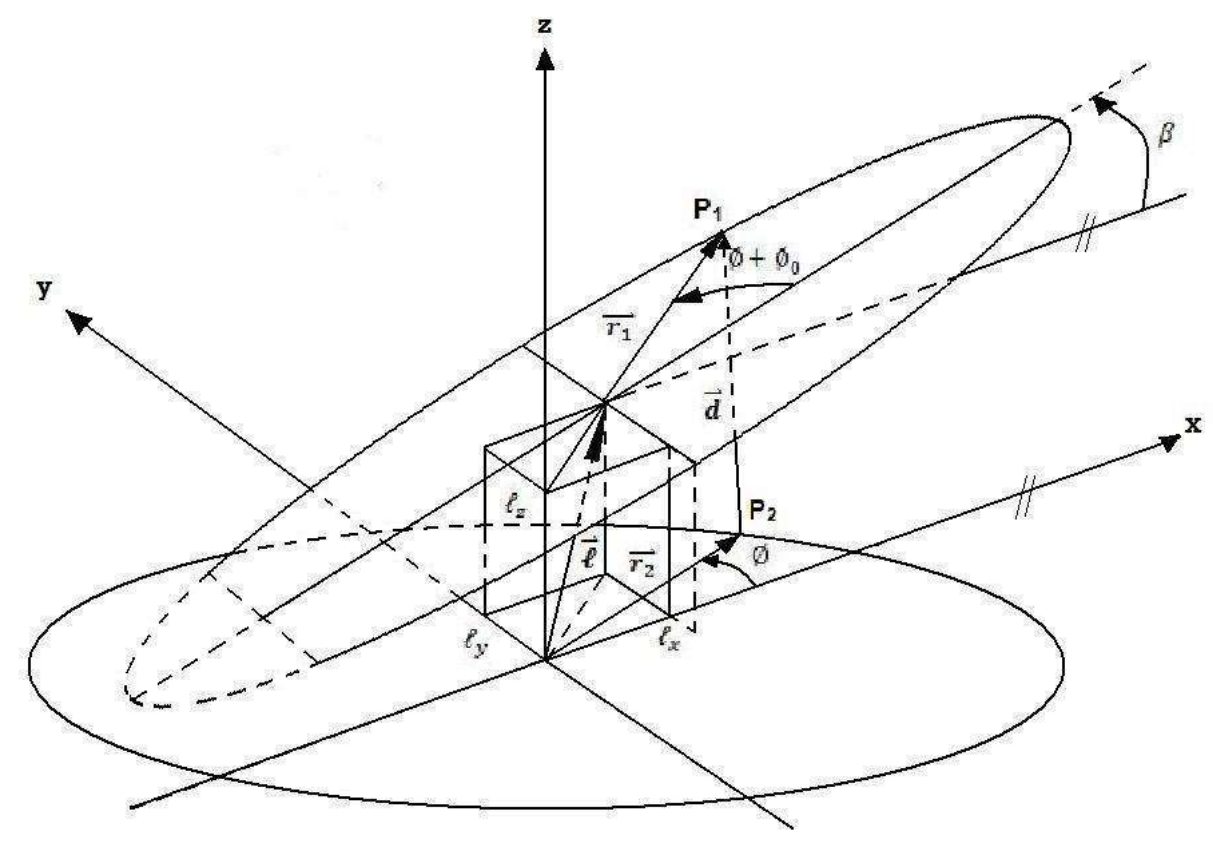

Figure 1 Distance Between Points $\mathbf{P}_{1}$ and $\mathbf{P}_{2}$ on Two Different Circles in Space $\left({ }^{4}\right)$ 


$$
\begin{aligned}
& \overrightarrow{\boldsymbol{r}}_{1}=\overrightarrow{\boldsymbol{r}}_{1}\left(\phi+\phi_{0}\right)=\hat{\boldsymbol{x}} r_{1} \operatorname{Cos}\left(\phi+\phi_{0}\right) \operatorname{Cos} \beta+\hat{\boldsymbol{y}} r_{1} \operatorname{Sin}\left(\phi+\phi_{0}\right)+\hat{\boldsymbol{z}} r_{1} \operatorname{Cos}\left(\phi+\phi_{0}\right) \operatorname{Sin} \beta \\
& \overrightarrow{\boldsymbol{r}}_{1}=\overrightarrow{\boldsymbol{r}}_{1}(\phi)=\left(\hat{\boldsymbol{x}} r_{1} \operatorname{Cos} \beta \operatorname{Cos} \phi_{0}+\hat{\boldsymbol{y}} r_{1} \operatorname{Sin} \phi_{0}+\hat{\boldsymbol{z}} r_{1} \operatorname{Sin} \beta \operatorname{Cos} \phi_{0}\right) \operatorname{Cos} \phi+ \\
& \left(-\hat{\boldsymbol{x}} r_{1} \operatorname{Cos} \beta \operatorname{Sin} \phi_{0}+\hat{\boldsymbol{y}} r_{1} \operatorname{Cos} \phi_{0}-\hat{\boldsymbol{z}} r_{1} \operatorname{Sin} \beta \operatorname{Sin} \phi_{0}\right) \operatorname{Sin} \phi \\
& \overrightarrow{\boldsymbol{r}}_{2}=\overrightarrow{\boldsymbol{r}}_{2}(\phi)=\hat{\boldsymbol{x}} r_{2} \operatorname{Cos} \phi+\hat{\boldsymbol{y}} r_{2} \operatorname{Sin} \phi \\
& \overrightarrow{\boldsymbol{r}}_{1} \cdot \overrightarrow{\boldsymbol{r}}_{1}=r_{1}^{2} \quad ; \quad\left|\overrightarrow{\boldsymbol{r}}_{1}\right|=r_{1}=\sqrt{\vec{r}_{1} \cdot \overrightarrow{\boldsymbol{r}}_{1}} \quad ; \quad \overrightarrow{\boldsymbol{r}}_{2} \cdot \overrightarrow{\boldsymbol{r}}_{2}=r_{2}^{2} \quad ; \quad\left|\overrightarrow{\boldsymbol{r}}_{2}\right|=r_{2}=\sqrt{\overrightarrow{\boldsymbol{r}}_{2} \cdot \overrightarrow{\boldsymbol{r}}_{2}} \\
& \vec{\ell}=\vec{\ell}(\phi)=\hat{\boldsymbol{x}} \ell_{x}(\phi)+\hat{\boldsymbol{y}} \ell_{y}(\phi)+\hat{\boldsymbol{z}} \ell_{z}(\phi) \\
& |\vec{\ell}(\phi)|=\ell(\phi)=\sqrt{\ell^{2}(\phi)}=\sqrt{\hat{\ell}(\phi) \cdot \vec{\ell}(\phi)}=\sqrt{\ell_{x}^{2}(\phi)+\ell_{y}{ }^{2}(\phi)+\ell_{z}^{2}(\phi)}
\end{aligned}
$$

The vector distance $\overrightarrow{\boldsymbol{d}}(\phi)$ (7) - (9) between any of these two points $\mathbf{P}_{1}$ phased at $\left(\phi+\phi_{0}\right)$ and $\mathbf{P}_{2}$ phased at $(\phi)$ on the two respective circles, and its magnitude $d(\phi)$ (10) which is also the scalar distance between $\mathbf{P}_{1}$ and $\mathbf{P}_{2}$, is as demonstrated in Figure $\mathbf{1}$ and expressed in the following vector equations in (7) - (10), based on (1) - (6). It is important to note that the scalar distance $d(\phi)(10)$ between $\mathbf{P}_{1}$ and $\mathbf{P}_{2}$ is calculated as the square root of $d^{2}(\phi)(10)$, which in turn is calculated in terms of the Dot $\operatorname{Product}^{1}[\overrightarrow{\boldsymbol{d}}(\phi) \cdot \overrightarrow{\boldsymbol{d}}(\phi)](10)$ of the vector distance $\overrightarrow{\boldsymbol{d}}(\phi)(7)$ - (9) with itself.

$$
\begin{gathered}
\overrightarrow{\boldsymbol{d}}(\phi)=\overrightarrow{\boldsymbol{r}}_{1}\left(\phi+\phi_{0}\right)-\overrightarrow{\boldsymbol{r}_{2}}(\phi)+\vec{\ell}(\phi)=\overrightarrow{\boldsymbol{r}_{1}}-\overrightarrow{\boldsymbol{r}_{2}}+\vec{\ell} \\
\overrightarrow{\boldsymbol{d}}(\phi)=\hat{\boldsymbol{x}} d_{x}(\phi)+\hat{\boldsymbol{y}} d_{y}(\phi)+\hat{\boldsymbol{z}} d_{z}(\phi) \Rightarrow\left\{\begin{array}{l}
d_{x}(\phi)=r_{1} \operatorname{Cos}\left(\phi+\phi_{0}\right) \operatorname{Cos} \beta-r_{2} \operatorname{Cos} \phi+\ell_{x}(\phi) \\
d_{y}(\phi)=r_{1} \operatorname{Sin}\left(\phi+\phi_{0}\right)-r_{2} \operatorname{Sin} \phi+\ell_{y}(\phi) \\
d_{z}(\phi)=r_{1} \operatorname{Cos}\left(\phi+\phi_{0}\right) \operatorname{Sin} \beta+\ell_{z}(\phi)
\end{array}\right. \\
\Rightarrow \overrightarrow{\boldsymbol{d}}(\phi)=\left[\hat{\boldsymbol{x}}\left(r_{1} \operatorname{Cos} \beta \operatorname{Cos} \phi_{0}-r_{2}\right)+\hat{\boldsymbol{y}} r_{1} \operatorname{Sin} \phi_{0}+\hat{\boldsymbol{z}} r_{1} \operatorname{Sin} \beta \operatorname{Cos} \phi_{0}\right] \operatorname{Cos} \phi+ \\
{\left[-\hat{\boldsymbol{x}} r_{1} \operatorname{Cos} \beta \operatorname{Sin} \phi_{0}+\hat{\boldsymbol{y}}\left(r_{1} \operatorname{Cos} \phi_{0}-r_{2}\right)-\hat{\boldsymbol{z}} r_{1} \operatorname{Sin} \beta \operatorname{Sin} \phi_{0}\right] \operatorname{Sin} \phi+} \\
{\left[\hat{\boldsymbol{x}} \ell_{x}(\phi)+\hat{\boldsymbol{y}} \ell_{y}(\phi)+\hat{\boldsymbol{z}} \ell_{z}(\phi)\right]} \\
d(\phi)=|\overrightarrow{\boldsymbol{d}}(\phi)|=\sqrt{d^{2}(\phi)}=\sqrt{\overrightarrow{\boldsymbol{d}}(\phi) \cdot \overrightarrow{\boldsymbol{d}}(\phi)}=\sqrt{\left[d_{x}(\phi)\right]^{2}+\left[d_{y}(\phi)\right]^{2}+\left[d_{z}(\phi)\right]^{2}}
\end{gathered}
$$


Vector distance $\overrightarrow{\boldsymbol{d}}(\phi)$ (9) at any value of the phase $\phi$, between points $\mathbf{P}_{1}$ phased at $\left(\phi+\phi_{0}\right)$ and $\mathbf{P}_{2}$ phased at $(\phi)$ on two respective circles, can equivalently be expressed as $\overrightarrow{\boldsymbol{d}}(\phi)$ (11) in terms of virtual vectors $\overrightarrow{\mathbf{X}}(\phi)$ (12) and $\overrightarrow{\mathbf{Y}}(\phi)$ (13), defined utilizing $\overrightarrow{\boldsymbol{r}}_{1}$ (1) - (2) and $\overrightarrow{\boldsymbol{r}}_{2}(3)$. Based on the definition of $\overrightarrow{\mathbf{X}}(\phi)$ (12) from (11), (5) and (9), the virtual vector $\overrightarrow{\boldsymbol{a}}$ (14) is also defined, and based on the definition of $\overrightarrow{\mathbf{Y}}(\phi)$ (13) from (11), (5) and (9), another virtual vector $\vec{b}$ (15) is also defined.

The magnitude $X(\phi)$ (12) of the virtual vector $\overrightarrow{\mathbf{X}}(\phi)$ (12) is calculated as the square root of $X^{2}(\phi)(12)$, which in turn is calculated in terms of the Dot $\operatorname{Product}^{1}[\overrightarrow{\mathbf{X}}(\phi) \cdot \overrightarrow{\mathbf{X}}(\phi)](12)$ of the vector $\overrightarrow{\mathbf{X}}(\phi)$ (12) with itself. The magnitude $Y(\phi)$ (13) of the virtual vector $\overrightarrow{\mathbf{Y}}(\phi)$ (13) is calculated as the square root of $Y^{2}(\phi)(13)$, which in turn is calculated in terms of the Dot Product $^{1}[\overrightarrow{\mathbf{Y}}(\phi) \cdot \overrightarrow{\mathbf{Y}}(\phi)](13)$ of the vector $\overrightarrow{\mathbf{Y}}(\phi)$ (13) with itself. The magnitude $a$ (16) of the virtual vector $\overrightarrow{\boldsymbol{a}}$ (14) is calculated as the square root of $a^{2}$ (16), which in turn is calculated in terms of the Dot $\operatorname{Product}^{1}(\overrightarrow{\boldsymbol{a}} \cdot \overrightarrow{\boldsymbol{a}})$ (16) of the vector $\overrightarrow{\boldsymbol{a}}$ (14) with itself. The magnitude $b$ (17) of the virtual vector $\overrightarrow{\boldsymbol{b}}(15)$ is calculated as the square root of $b^{2}(17)$, which in turn is calculated in terms of the Dot $\operatorname{Product}^{1}(\overrightarrow{\boldsymbol{b}} \cdot \overrightarrow{\boldsymbol{b}})(17)$ of the vector $\overrightarrow{\boldsymbol{b}}$ (15) with itself. When the phase difference $\phi_{0}(1)$ is constant for all $\phi$, magnitudes $a$ (16) and $b$ (17) are constant for all $\phi$, and when the phase difference $\phi_{0}(1)$ is time $(t)$-dependent, i.e. if $\phi_{0}=\phi_{0}(t), a$ (16) and $b(17)$ are also time $(t)$-dependent for different $\phi$. Based on the definitions of $\overrightarrow{\boldsymbol{a}}(14)$ and $\overrightarrow{\boldsymbol{b}}(15)$, their $\operatorname{Dot}_{\operatorname{Product}^{1}}(\overrightarrow{\boldsymbol{a}} \cdot \overrightarrow{\boldsymbol{b}})$ (18) is also defined.

$$
\begin{gathered}
\overrightarrow{\boldsymbol{d}}(\phi)=\overrightarrow{\mathbf{X}}(\phi)+\overrightarrow{\mathbf{Y}}(\phi)+\vec{\ell}(\phi) \quad \text { where } \quad \overrightarrow{\boldsymbol{r}_{1}}(\phi)-\overrightarrow{\boldsymbol{r}_{2}}(\phi)=\overrightarrow{\mathbf{X}}(\phi)+\overrightarrow{\mathbf{Y}}(\phi)=\overrightarrow{\boldsymbol{a}} \operatorname{Cos} \phi+\overrightarrow{\boldsymbol{b}} \operatorname{Sin} \phi \\
\overrightarrow{\mathbf{X}}(\phi)=\overrightarrow{\boldsymbol{a}} \operatorname{Cos} \phi \quad ; \quad \overrightarrow{\mathbf{X}}(\phi) \cdot \overrightarrow{\mathbf{X}}(\phi)=X^{2}(\phi)=\overrightarrow{\boldsymbol{a}} \cdot \overrightarrow{\boldsymbol{a}} \operatorname{Cos}^{2} \phi=a^{2} \operatorname{Cos}^{2} \phi \quad ;|\overrightarrow{\mathbf{X}}(\phi)|=X(\phi) \\
\overrightarrow{\mathbf{Y}}(\phi)=\overrightarrow{\boldsymbol{b}} \operatorname{Sin} \phi \quad ; \quad \overrightarrow{\mathbf{Y}}(\phi) \cdot \overrightarrow{\mathbf{Y}}(\phi)=Y^{2}(\phi)=\overrightarrow{\boldsymbol{b}} \cdot \overrightarrow{\boldsymbol{b}} \operatorname{Sin}^{2} \phi=b^{2} \operatorname{Sin}^{2} \phi \quad ;|\overrightarrow{\mathbf{Y}}(\phi)|=Y(\phi) \\
\overrightarrow{\boldsymbol{a}}=\hat{\boldsymbol{x}}\left(r_{1} \operatorname{Cos} \beta \operatorname{Cos} \phi_{0}-r_{2}\right)+\hat{\boldsymbol{y}} r_{1} \operatorname{Sin} \phi_{0}+\hat{\boldsymbol{z}} r_{1} \operatorname{Sin} \beta \operatorname{Cos} \phi_{0} \Rightarrow \overrightarrow{\boldsymbol{a}}=\left[\overrightarrow{\boldsymbol{r}_{1}}(\phi)-\overrightarrow{\boldsymbol{r}_{2}}(\phi)\right](\phi=0) \\
\overrightarrow{\boldsymbol{b}}=-\hat{\boldsymbol{x}} r_{1} \operatorname{Cos} \beta \operatorname{Sin} \phi_{0}+\hat{\boldsymbol{y}}\left(r_{1} \operatorname{Cos} \phi_{0}-r_{2}\right)-\hat{\boldsymbol{z}} r_{1} \operatorname{Sin} \beta \operatorname{Sin} \phi_{0} \Rightarrow \overrightarrow{\boldsymbol{b}}=\left[\overrightarrow{\boldsymbol{r}_{1}}(\phi)-\overrightarrow{\boldsymbol{r}_{2}}(\phi)\right]\left(\phi=\frac{\pi}{2}\right)
\end{gathered}
$$




$$
\begin{gathered}
\overrightarrow{\boldsymbol{a}} \cdot \overrightarrow{\boldsymbol{a}}=a^{2}=r_{1}^{2}-2 r_{1} r_{2} \operatorname{Cos} \beta \operatorname{Cos} \phi_{0}+r_{2}^{2} ;|\overrightarrow{\boldsymbol{a}}|=a \\
\overrightarrow{\boldsymbol{b}} \cdot \overrightarrow{\boldsymbol{b}}=b^{2}=r_{1}^{2}-2 r_{1} r_{2} \operatorname{Cos} \phi_{0}+r_{2}^{2} ; \quad|\overrightarrow{\boldsymbol{b}}|=b \\
\overrightarrow{\boldsymbol{a}} \cdot \overrightarrow{\boldsymbol{b}}=r_{1} r_{2}(\operatorname{Cos} \beta-1) \operatorname{Sin} \phi_{0}
\end{gathered}
$$

According to the definitions of vectors $\overrightarrow{\mathbf{X}}(\phi)$ (12), $\overrightarrow{\mathbf{Y}}(\phi)$ (13), $\overrightarrow{\boldsymbol{a}}$ (14), and $\overrightarrow{\boldsymbol{b}}$ (15), as described in (11) - (17), the relation in (19) is valid and holds for all $\phi$.

$$
\begin{aligned}
& \frac{\overrightarrow{\mathbf{X}}(\phi) \cdot \overrightarrow{\mathbf{X}}(\phi)}{\overrightarrow{\boldsymbol{a}} \cdot \overrightarrow{\boldsymbol{a}}}+\frac{\overrightarrow{\mathbf{Y}}(\phi) \cdot \overrightarrow{\mathbf{Y}}(\phi)}{\overrightarrow{\boldsymbol{b}} \cdot \overrightarrow{\boldsymbol{b}}}=\frac{X^{2}(\phi)}{a^{2}}+\frac{Y^{2}(\phi)}{b^{2}}=\operatorname{Cos}^{2} \phi+\operatorname{Sin}^{2} \phi=1 \\
\Rightarrow & \frac{\overrightarrow{\mathbf{X}}(\phi) \cdot \overrightarrow{\mathbf{X}}(\phi)}{\overrightarrow{\boldsymbol{a}} \cdot \overrightarrow{\boldsymbol{a}}}+\frac{\overrightarrow{\mathbf{Y}}(\phi) \cdot \overrightarrow{\mathbf{Y}}(\phi)}{\overrightarrow{\boldsymbol{b}} \cdot \overrightarrow{\boldsymbol{b}}}=1 \quad\left(\begin{array}{l}
\text { Definition of Vector Ellipse } \\
\text { in Multi-Dimensions }
\end{array}\right) \\
\Rightarrow & \frac{X^{2}(\phi)}{a^{2}+\frac{Y^{2}(\phi)}{b^{2}}=1 \quad \text { (Definition of Scalar Ellipse in 2-Dimensions) }}
\end{aligned}
$$

Therefore, the relation in (19) reveals the validity of (20) and (21) for the vector pair $[\overrightarrow{\mathbf{X}}(\phi), \overrightarrow{\mathbf{Y}}(\phi)](12)$ - (13) and its magnitude pair $[X(\phi), Y(\phi)](12)$ - (13), respectively. As

(21) is the defining equation of an ellipse ${ }^{2}$ in two dimensions, where $a$ (16) is semi-major ${ }^{3}$ axis and $b(17)$ is semi-minor ${ }^{3}$ axis of the ellipse ${ }^{2}$ when $(a>b)^{2,3}$ holds, and vice versa, with (21) reducing to the special case of a circle when $(a=b)^{2,3}$ holds, we can claim that (20) indicates that the vector pair $[\overrightarrow{\mathbf{X}}(\phi), \overrightarrow{\mathbf{Y}}(\phi)](12)$ - (13) defines points on a vector ellipse in three dimensions in the most general case. In other words, the vector distance $\overrightarrow{\boldsymbol{d}}(\phi)$ (9) between two points $\mathbf{P}_{1}$ phased at $\left(\phi+\phi_{0}\right)$ and $\mathbf{P}_{2}$ phased at $(\phi)$ on two respective circles, whose centers are displaced by a constant or variable vector $\vec{\ell}(\phi)$ (5), can equivalently be mathematically expressed and interpreted as the distance $\overrightarrow{\boldsymbol{d}}(\phi)$ (11) of points on a virtual vector ellipse, whose locations with respect to a virtual origin at each $\phi$ are determined by the sum of vector pair $[\overrightarrow{\mathbf{X}}(\phi), \overrightarrow{\mathbf{Y}}(\phi)]$ (12) - (13), from another fixed or moving point displaced from the same virtual origin of the ellipse by a constant or variable vector $[-\vec{\ell}(\phi)](5)$, where $\overrightarrow{\boldsymbol{a}}(14)$ and $\overrightarrow{\boldsymbol{b}}$ (15) are fixed or variable semi-major ${ }^{3}$ and semi-minor ${ }^{3}$ 
axis vectors of the vector ellipse. This result is mathematically valid even when the phase difference $\phi_{0}$ (1) is a variable function of time $(t)$, i.e. even if $\phi_{0}=\phi_{0}(t)$. This revelation is the first finding presented in this Article, where we have also mathematically introduced the concept of a "vector ellipse" (20) in three-dimensional space based on our analysis introduced along the lines of (1) - (21).

The meaning of this mathematical finding in physics is that moving points on two circles in space vector-wise mimic an elliptical path with respect to each other, whether they are moving with the same angular velocity, or different but fixed angular velocities, or even with different and changing angular velocities with respect to their own centers of revolution, virtually seeing each other as positioned at a instantaneously stationary point in space on their respective virtual ecliptic plane. In the book "Everyhing Is A Circle: A New Model For Orbits Of Bodies In The Universe" 4 , this finding is used as a basis to analyze the orbits of the Sun $^{6}$, the Earth ${ }^{7,8}$, and the Moon ${ }^{9}$, based on the relative cyclic distances between them, also proposing a new Circular Orbital Model for moving bodies in the Universe with some observational proof.

Square of focal ${ }^{2,4}$ distance $\left(c^{2}\right)(22)$ of the vector ellipse (20) can be found using (16) - (17), and its eccentricity ${ }^{2} e(23)$ can be determined using (16) - (17) and (22).

$$
\begin{gathered}
c^{2}=\left|a^{2}-b^{2}\right|=2 r_{1} r_{2}(1-\operatorname{Cos} \beta)\left|\operatorname{Cos} \phi_{0}\right| \quad(\text { Focal Distance Squared }) \\
e^{(a>b)}=\frac{c}{a}=\sqrt{\frac{2 r_{1} r_{2}(1-\operatorname{Cos} \beta)\left|\operatorname{Cos} \phi_{0}\right|}{r_{1}^{2}-2 r_{1} r_{2} \operatorname{Cos} \beta \operatorname{Cos} \phi_{0}+r_{2}^{2}}} \quad \text { or } \quad e \stackrel{(a<b)}{=} \frac{c}{b}=\sqrt{\frac{2 r_{1} r_{2}(1-\operatorname{Cos} \beta)\left|\operatorname{Cos} \phi_{0}\right|}{r_{1}^{2}-2 r_{1} r_{2} \operatorname{Cos} \phi_{0}+r_{2}^{2}}}
\end{gathered}
$$

Note that $\overrightarrow{\boldsymbol{a}}$ (14) is the vector value of $\left[\overrightarrow{\boldsymbol{r}_{1}}(\phi)-\overrightarrow{\boldsymbol{r}_{2}}(\phi)\right](11)$ when $(\phi=0)$, and $\overrightarrow{\boldsymbol{b}}$ (15) is the vector value of $\left[\overrightarrow{\boldsymbol{r}}_{1}(\phi)-\overrightarrow{\boldsymbol{r}}_{2}(\phi)\right](11)$ when $\left(\phi=\frac{\pi}{2}\right)$. Throughout a respective cycle of points $\mathbf{P}_{1}$ and $\mathbf{P}_{2}$ moving around their own circles, $\mathbf{P}_{1}$ phased at $\left(\phi+\phi_{0}\right)$ and $\mathbf{P}_{2}$ phased at $(\phi)$, the $\left[\overrightarrow{\boldsymbol{r}_{1}}(\phi)-\overrightarrow{\boldsymbol{r}_{2}}(\phi)\right](11)$ vector has a value of $(\overrightarrow{\boldsymbol{a}} \operatorname{Cos} \phi+\overrightarrow{\boldsymbol{b}} \operatorname{Sin} \phi)(11)$ at each phase $\phi$, and moves in the plane formed by the $\overrightarrow{\boldsymbol{a}}$ (14) and $\overrightarrow{\boldsymbol{b}}$ (15) vectors, namely the $\overrightarrow{\boldsymbol{a}}-\overrightarrow{\boldsymbol{b}}$ plane, which is a variable moving plane in three dimensions if $\phi_{0}$ is a variable function of time $t$, i.e. if $\phi_{0}=\phi_{0}(t)$, and a fixed plane otherwise. 
We now continue to analyze the situation of the distance between points $\mathbf{P}_{1}$ and $\mathbf{P}_{2}$ on two circles in multiple dimensions.

Consider a system of two circles in four-dimensions defined in terms of perpendicular coordinates $\left(\hat{\boldsymbol{u}}_{1}, \hat{\boldsymbol{u}}_{2}, \hat{\boldsymbol{u}}_{3}, \hat{\boldsymbol{u}}_{4}\right)$. In this case, the two circles can be defined in the simplest case to have vector radii $\overrightarrow{\boldsymbol{r}}_{1}(24)$ - (25) and $\overrightarrow{\boldsymbol{r}}_{2}$ (26), with constant magnitudes $r_{1}(27)$ and $r_{2}(27)$, respectively, which are radius vectors at point $\mathbf{P}_{1}$ phased at $\left(\phi+\phi_{0}\right)$ and $\mathbf{P}_{2}$ phased at $(\phi)$ on the two circles, respectively, phased apart by a constant or time $(t)$-dependent angle $\phi_{0}(24)$. Note that $\beta_{1}(24)-(25)$ is taken to be the constant inclination angle between these two circles in the $\hat{\boldsymbol{u}}_{1}-\hat{\boldsymbol{u}}_{3}$ dimension plane, and $\beta_{2}(24)-(25)$ is taken to be the constant inclination angle between these two circles in the $\hat{\boldsymbol{u}}_{2}-\hat{\boldsymbol{u}}_{4}$ dimension plane.

$$
\begin{aligned}
& \overrightarrow{\boldsymbol{r}}_{1}=\overrightarrow{\boldsymbol{r}}_{1}\left(\phi+\phi_{0}\right)=\hat{\boldsymbol{u}}_{1} r_{1} \operatorname{Cos}\left(\phi+\phi_{0}\right) \operatorname{Cos} \beta_{1}+\hat{\boldsymbol{u}}_{2} r_{1} \operatorname{Sin}\left(\phi+\phi_{0}\right) \operatorname{Cos} \beta_{2} \\
& +\hat{\boldsymbol{u}}_{3} r_{1} \operatorname{Cos}\left(\phi+\phi_{0}\right) \operatorname{Sin} \beta_{1}+\hat{\boldsymbol{u}}_{4} r_{1} \operatorname{Sin}\left(\phi+\phi_{0}\right) \operatorname{Sin} \beta_{2} \\
& \overrightarrow{\boldsymbol{r}}_{1}=\overrightarrow{\boldsymbol{r}}_{1}(\phi)=\left(\hat{\boldsymbol{u}}_{1} r_{1} \operatorname{Cos} \beta_{1} \operatorname{Cos} \phi_{0}+\hat{\boldsymbol{u}}_{2} r_{1} \operatorname{Cos} \beta_{2} \operatorname{Sin} \phi_{0}+\hat{\boldsymbol{u}}_{3} r_{1} \operatorname{Sin} \beta_{1} \operatorname{Cos} \phi_{0}+\hat{\boldsymbol{u}}_{4} r_{1} \operatorname{Sin} \beta_{2} \operatorname{Sin} \phi_{0}\right) \operatorname{Cos} \phi \\
& +\left(-\hat{\boldsymbol{u}}_{1} r_{1} \operatorname{Cos} \beta_{1} \operatorname{Sin} \phi_{0}+\hat{\boldsymbol{u}}_{2} r_{1} \operatorname{Cos} \beta_{2} \operatorname{Cos} \phi_{0}-\hat{\boldsymbol{u}}_{3} r_{1} \operatorname{Sin} \beta_{1} \operatorname{Sin} \phi_{0}+\hat{\boldsymbol{u}}_{4} r_{1} \operatorname{Sin} \beta_{2} \operatorname{Cos} \phi_{0}\right) \operatorname{Sin} \phi \\
& \overrightarrow{\boldsymbol{r}}_{2}=\overrightarrow{\boldsymbol{r}}_{2}(\phi)=\hat{\boldsymbol{u}}_{1} r_{2} \operatorname{Cos} \phi+\hat{\boldsymbol{u}}_{2} r_{2} \operatorname{Sin} \phi \\
& \overrightarrow{\boldsymbol{r}}_{1} \cdot \overrightarrow{\boldsymbol{r}}_{1}=r_{1}^{2} \quad ; \quad\left|\overrightarrow{\boldsymbol{r}}_{1}\right|=r_{1}=\sqrt{\overrightarrow{\boldsymbol{r}}_{1} \cdot \overrightarrow{\boldsymbol{r}}_{1}} \quad ; \quad \overrightarrow{\boldsymbol{r}}_{2} \cdot \overrightarrow{\boldsymbol{r}}_{2}=r_{2}^{2} \quad ; \quad\left|\overrightarrow{\boldsymbol{r}}_{2}\right|=r_{2}=\sqrt{\overrightarrow{\boldsymbol{r}}_{2} \cdot \overrightarrow{\boldsymbol{r}}_{2}}
\end{aligned}
$$

The centers of these two circles in four-dimensions are displaced by a constant or variable vector $\vec{\ell}(\phi)$ (28) with magnitude $\ell(\phi)$ (29) at each constant or variable phase $\phi$, defined in terms of perpendicular coordinates $\left(\hat{\boldsymbol{u}}_{1}, \hat{\boldsymbol{u}}_{2}, \hat{\boldsymbol{u}}_{3}, \hat{\boldsymbol{u}}_{4}\right)$.

$$
\begin{gathered}
\vec{\ell}=\vec{\ell}(\phi)=\hat{\boldsymbol{u}}_{1} \ell_{u_{1}}(\phi)+\hat{\boldsymbol{u}}_{2} \ell_{u_{2}}(\phi)+\hat{\boldsymbol{u}}_{3} \ell_{u_{3}}(\phi)+\hat{\boldsymbol{u}}_{4} \ell_{u_{4}}(\phi) \\
|\vec{\ell}(\phi)|=\ell(\phi)=\sqrt{\ell^{2}(\phi)}=\sqrt{\hat{\ell}(\phi) \cdot \vec{\ell}(\phi)}=\sqrt{\ell_{u_{1}}{ }^{2}(\phi)+\ell_{u_{2}}{ }^{2}(\phi)+\ell_{u_{3}}{ }^{2}(\phi)+\ell_{u_{4}}{ }^{2}(\phi)}
\end{gathered}
$$

Similar to the case in three dimensions for $\overrightarrow{\boldsymbol{d}}(\phi)(11)$, the vector distance between points $\mathbf{P}_{1}$ phased at $\left(\phi+\phi_{0}\right)$ and $\mathbf{P}_{2}$ phased at $(\phi)$ on the two respective circles in four dimensions, at any value of the phase $\phi$, can be equivalently expressed as $\overrightarrow{\boldsymbol{d}}(\phi)$ (30) in terms of virtual vectors $\overrightarrow{\mathbf{X}}(\phi)$ (31) and $\overrightarrow{\mathbf{Y}}(\phi)$ (32), defined utilizing $\overrightarrow{\boldsymbol{r}}_{1}(24)$ - (25) and $\overrightarrow{\boldsymbol{r}}_{2}$ (26). 


$$
\begin{aligned}
& \overrightarrow{\boldsymbol{d}}(\phi)=\overrightarrow{\mathbf{X}}(\phi)+\overrightarrow{\mathbf{Y}}(\phi)+\vec{\ell}(\phi) \text { where } \quad \overrightarrow{\boldsymbol{r}_{1}}(\phi)-\overrightarrow{\boldsymbol{r}_{2}}(\phi)=\overrightarrow{\mathbf{X}}(\phi)+\overrightarrow{\mathbf{Y}}(\phi)=\overrightarrow{\boldsymbol{a}} \operatorname{Cos} \phi+\overrightarrow{\boldsymbol{b}} \operatorname{Sin} \phi \\
& \overrightarrow{\mathbf{X}}(\phi)=\overrightarrow{\boldsymbol{a}} \operatorname{Cos} \phi \quad ; \quad \overrightarrow{\mathbf{X}}(\phi) \cdot \overrightarrow{\mathbf{X}}(\phi)=X^{2}(\phi)=\overrightarrow{\boldsymbol{a}} \cdot \overrightarrow{\boldsymbol{a}} \operatorname{Cos}^{2} \phi=a^{2} \operatorname{Cos}^{2} \phi \quad ; \quad|\overrightarrow{\mathbf{X}}(\phi)|=X(\phi) \\
& \overrightarrow{\mathbf{Y}}(\phi)=\overrightarrow{\boldsymbol{b}} \operatorname{Sin} \phi \quad ; \quad \overrightarrow{\mathbf{Y}}(\phi) \cdot \overrightarrow{\mathbf{Y}}(\phi)=Y^{2}(\phi)=\overrightarrow{\boldsymbol{b}} \cdot \overrightarrow{\boldsymbol{b}} \operatorname{Sin}^{2} \phi=b^{2} \operatorname{Sin}^{2} \phi \quad ; \quad|\overrightarrow{\mathbf{Y}}(\phi)|=Y(\phi) \\
& \overrightarrow{\boldsymbol{a}}=\hat{\boldsymbol{u}}_{1}\left(r_{1} \operatorname{Cos} \beta_{1} \operatorname{Cos} \phi_{0}-r_{2}\right)+\hat{\boldsymbol{u}}_{2} r_{1} \operatorname{Cos} \beta_{2} \operatorname{Sin} \phi_{0}+\hat{\boldsymbol{u}}_{3} r_{1} \operatorname{Sin} \beta_{1} \operatorname{Cos} \phi_{0}+\hat{\boldsymbol{u}}_{4} r_{1} \operatorname{Sin} \beta_{2} \operatorname{Sin} \phi_{0} \\
& \Rightarrow \overrightarrow{\boldsymbol{a}}=\left[\overrightarrow{\boldsymbol{r}_{1}}(\phi)-\overrightarrow{\boldsymbol{r}_{2}}(\phi)\right](\phi=0) \\
& \overrightarrow{\boldsymbol{b}}=-\hat{\boldsymbol{u}}_{1} r_{1} \operatorname{Cos} \beta_{1} \operatorname{Sin} \phi_{0}+\hat{\boldsymbol{u}}_{2}\left(r_{1} \operatorname{Cos} \beta_{2} \operatorname{Cos} \phi_{0}-r_{2}\right)-\hat{\boldsymbol{u}}_{3} r_{1} \operatorname{Sin} \beta_{1} \operatorname{Sin} \phi_{0}+\hat{\boldsymbol{u}}_{4} r_{1} \operatorname{Sin} \beta_{2} \operatorname{Cos} \phi_{0} \\
& \Rightarrow \vec{b}=\left[\vec{r}_{1}(\phi)-\overrightarrow{r_{2}}(\phi)\right]\left(\phi=\frac{\pi}{2}\right) \\
& \overrightarrow{\boldsymbol{a}} \cdot \overrightarrow{\boldsymbol{a}}=a^{2}=r_{1}^{2}-2 r_{1} r_{2} \operatorname{Cos} \beta_{1} \operatorname{Cos} \phi_{0}+r_{2}^{2} \quad ; \quad|\overrightarrow{\boldsymbol{a}}|=a \\
& \overrightarrow{\boldsymbol{b}} \cdot \overrightarrow{\boldsymbol{b}}=b^{2}=r_{1}^{2}-2 r_{1} r_{2} \operatorname{Cos} \beta_{2} \operatorname{Cos} \phi_{0}+r_{2}^{2} \quad ; \quad|\overrightarrow{\boldsymbol{b}}|=b \\
& \overrightarrow{\boldsymbol{a}} \cdot \overrightarrow{\boldsymbol{b}}=r_{1} r_{2}\left(\operatorname{Cos} \beta_{1}-\operatorname{Cos} \beta_{2}\right) \operatorname{Sin} \phi_{0}
\end{aligned}
$$

Based on the definition of $\overrightarrow{\mathbf{X}}(\phi)$ (31) from (30) and the definition of $\overrightarrow{\mathbf{Y}}(\phi)$ (32) from (30), $\overrightarrow{\boldsymbol{a}}$ (33) and $\overrightarrow{\boldsymbol{b}}$ (34) vectors can be defined, which turn out to be semi-major ${ }^{3}$ and semi-minor ${ }^{3}$ axis vectors of the relative ellipse ${ }^{2}$ formed based on the distance $\overrightarrow{\boldsymbol{d}}(\phi)$ (30) between points $\mathbf{P}_{1}$ and $\mathbf{P}_{2}$, with magnitudes $a$ (35) and $b$ (36), respectively, and $\operatorname{Dot}_{\operatorname{Product}^{1}}(\overrightarrow{\boldsymbol{a}} \cdot \overrightarrow{\boldsymbol{b}})$ (37). According to the definitions of vectors $\overrightarrow{\mathbf{X}}(\phi)$ (31), $\overrightarrow{\mathbf{Y}}(\phi)$ (32), $\overrightarrow{\boldsymbol{a}}$ (33), and $\overrightarrow{\boldsymbol{b}}$ (34), as described in (30) - (37), the relation in (19) is valid and holds for all $\phi$. Thus, the relation in (19) reveals the validity of (20) and (21) for the vector pair $[\overrightarrow{\mathbf{X}}(\phi), \overrightarrow{\mathbf{Y}}(\phi)](31)$ - (32) and their magnitude pair $[X(\phi), Y(\phi)](31)$ - (32), respectively. As (21) is the defining equation of an ellipse ${ }^{2}$ in two dimensions, where $a$ (35) is the semi-major ${ }^{3}$ axis and $b$ (36) is the semi-minor $^{3}$ axis of the ellipse ${ }^{2}$ when $(a>b)^{2,3}$ holds, and vice versa, with (21) reducing to the special case of a circle when $(a=b)^{2,3}$ holds, we can claim that (20) indicates that the vector pair $[\overrightarrow{\mathbf{X}}(\phi), \overrightarrow{\mathbf{Y}}(\phi)](31)$ - (32) defines points on a vector ellipse in four dimensions in the most general case. In other words, the vector distance $\overrightarrow{\boldsymbol{d}}(\phi)$ (30) between two points $\mathbf{P}_{1}$ 
phased at $\left(\phi+\phi_{0}\right)$ and $\mathbf{P}_{2}$ phased at $(\phi)$ on two respective circles in four dimensions, whose centers are displaced by a constant or variable vector $\vec{\ell}(\phi)$ (28), can equivalently be mathematically expressed and interpreted as distance of points on a virtual vector ellipse, whose locations with respect to a virtual origin at each $\phi$ are determined by the sum of vector pair $[\overrightarrow{\mathbf{X}}(\phi), \overrightarrow{\mathbf{Y}}(\phi)]$ (31) - (32), from another fixed or moving point displaced from the same virtual origin of the ellipse by a constant or variable vector $[-\vec{\ell}(\phi)](28), \overrightarrow{\boldsymbol{a}}$ (33) and $\overrightarrow{\boldsymbol{b}}$ (34) being the fixed or variable semi-major ${ }^{3}$ and semi-minor ${ }^{3}$ axis vectors of the vector ellipse. This result is mathematically valid even when the phase difference $\phi_{0}$ is a variable function of time $(t)$, i.e. even if $\phi_{0}=\phi_{0}(t)$. This is a finding presented in this Article for two circles in four dimensions.

Square of the focal ${ }^{2,4}$ distance $\left(c^{2}\right)(38)$ of this vector ellipse ${ }^{2}$ can be found using (35) - (36), and its eccentricity ${ }^{2} e(39)$ can be determined using (35) - (36) and (38).

$$
\begin{gathered}
c^{2}=\left|a^{2}-b^{2}\right|=2 r_{1} r_{2}\left(\operatorname{Cos} \beta_{2}-\operatorname{Cos} \beta_{1}\right)\left|\operatorname{Cos} \phi_{0}\right| \quad(\text { Focal Distance Squared }) \\
e^{(a>b)}=\frac{c}{a}=\sqrt{\frac{2 r_{1} r_{2}\left(\operatorname{Cos} \beta_{2}-\operatorname{Cos} \beta_{1}\right)\left|\operatorname{Cos} \phi_{0}\right|}{r_{1}^{2}-2 r_{1} r_{2} \operatorname{Cos} \beta_{1} \operatorname{Cos} \phi_{0}+r_{2}^{2}}} \text { or } \quad e^{(a<b)}=\frac{c}{b}=\sqrt{\frac{2 r_{1} r_{2}\left(\operatorname{Cos} \beta_{2}-\operatorname{Cos} \beta_{1}\right)\left|\operatorname{Cos} \phi_{0}\right|}{r_{1}^{2}-2 r_{1} r_{2} \operatorname{Cos} \beta_{2} \operatorname{Cos} \phi_{0}+r_{2}^{2}}}
\end{gathered}
$$

Consider a system of two circles in five dimensions defined in terms of perpendicular coordinates $\left(\hat{\boldsymbol{u}}_{1}, \hat{\boldsymbol{u}}_{2}, \hat{\boldsymbol{u}}_{3}, \hat{\boldsymbol{u}}_{4}, \hat{\boldsymbol{u}}_{5}\right)$. In this case, the two circles can be defined in the simplest case to have vector radii $\vec{r}_{1}(40)$ - (41) and $\vec{r}_{2}$ (42), with constant magnitudes $r_{1}$ (43) and $r_{2}(43)$, respectively, which are radius vectors at point $\mathbf{P}_{1}$ phased at $\left(\phi+\phi_{0}\right)$ and $\mathbf{P}_{2}$ phased at $(\phi)$ on the two circles, respectively, phased apart by a constant or time $(t)$-dependent angle $\phi_{0}(40)$. Note that $\beta_{1}(40)-(41)$ is taken to be the constant inclination angle between these two circles in the $\hat{\boldsymbol{u}}_{1}-\hat{\boldsymbol{u}}_{3}$ dimension plane, $\beta_{2}(40)-(41)$ is taken to be the constant inclination angle between these two circles in the $\hat{\boldsymbol{u}}_{2}-\hat{\boldsymbol{u}}_{4}$ dimension plane, and $\beta_{3}(40)-(41)$ is taken to be the constant inclination angle of these two circles between the $\hat{\boldsymbol{u}}_{1}-\hat{\boldsymbol{u}}_{3}$ dimension plane and the $\hat{\boldsymbol{u}}_{5}$ dimension. 


$$
\begin{aligned}
& \overrightarrow{\boldsymbol{r}}_{1}=\overrightarrow{\boldsymbol{r}}_{1}\left(\phi+\phi_{0}\right)=\hat{\boldsymbol{u}}_{1} r_{1} \operatorname{Cos}\left(\phi+\phi_{0}\right) \operatorname{Cos} \beta_{1} \operatorname{Cos} \beta_{3}+\hat{\boldsymbol{u}}_{2} r_{1} \operatorname{Sin}\left(\phi+\phi_{0}\right) \operatorname{Cos} \beta_{2} \\
& +\hat{\boldsymbol{u}}_{3} r_{1} \operatorname{Cos}\left(\phi+\phi_{0}\right) \operatorname{Sin} \beta_{1} \operatorname{Cos} \beta_{3}+\hat{\boldsymbol{u}}_{4} r_{1} \operatorname{Sin}\left(\phi+\phi_{0}\right) \operatorname{Sin} \beta_{2}+\hat{\boldsymbol{u}}_{5} r_{1} \operatorname{Cos}\left(\phi+\phi_{0}\right) \operatorname{Sin} \beta_{3} \\
& \overrightarrow{\boldsymbol{r}}_{1}=\overrightarrow{\boldsymbol{r}}_{1}(\phi)=\left(\begin{array}{l}
\hat{\boldsymbol{u}}_{1} r_{1} \operatorname{Cos} \beta_{1} \operatorname{Cos} \beta_{3} \operatorname{Cos} \phi_{0}+\hat{\boldsymbol{u}}_{2} r_{1} \operatorname{Cos} \beta_{2} \operatorname{Sin} \phi_{0}+\hat{\boldsymbol{u}}_{3} r_{1} \operatorname{Sin} \beta_{1} \operatorname{Cos} \beta_{3} \operatorname{Cos} \phi_{0} \\
+\hat{\boldsymbol{u}}_{4} r_{1} \operatorname{Sin} \beta_{2} \operatorname{Sin} \phi_{0}+\hat{\boldsymbol{u}}_{5} r_{1} \operatorname{Sin} \beta_{3} \operatorname{Cos} \phi_{0}
\end{array}\right) \operatorname{Cos} \phi+ \\
& \left(\begin{array}{l}
-\hat{\boldsymbol{u}}_{1} r_{1} \operatorname{Cos} \beta_{1} \operatorname{Cos} \beta_{3} \operatorname{Sin} \phi_{0}+\hat{\boldsymbol{u}}_{2} r_{1} \operatorname{Cos} \beta_{2} \operatorname{Cos} \phi_{0}-\hat{\boldsymbol{u}}_{3} r_{1} \operatorname{Sin} \beta_{1} \operatorname{Cos} \beta_{3} \operatorname{Sin} \phi_{0} \\
+\hat{\boldsymbol{u}}_{4} r_{1} \operatorname{Sin} \beta_{2} \operatorname{Cos} \phi_{0}-\hat{\boldsymbol{u}}_{5} r_{1} \operatorname{Sin} \beta_{3} \operatorname{Sin} \phi_{0}
\end{array}\right) \operatorname{Sin} \phi \\
& \overrightarrow{\boldsymbol{r}}_{2}=\overrightarrow{\boldsymbol{r}}_{2}(\phi)=\hat{\boldsymbol{u}}_{1} r_{2} \operatorname{Cos} \phi+\hat{\boldsymbol{u}}_{2} r_{2} \operatorname{Sin} \phi \\
& \overrightarrow{\boldsymbol{r}}_{1} \cdot \overrightarrow{\boldsymbol{r}}_{1}=r_{1}^{2} \quad ; \quad\left|\overrightarrow{\boldsymbol{r}}_{1}\right|=r_{1}=\sqrt{\overrightarrow{\boldsymbol{r}}_{1} \cdot \overrightarrow{\boldsymbol{r}}_{1}} \quad ; \quad \overrightarrow{\boldsymbol{r}}_{2} \cdot \overrightarrow{\boldsymbol{r}}_{2}=r_{2}^{2} \quad ; \quad\left|\overrightarrow{\boldsymbol{r}}_{2}\right|=r_{2}=\sqrt{\overrightarrow{\boldsymbol{r}}_{2} \cdot \overrightarrow{\boldsymbol{r}}_{2}}
\end{aligned}
$$

The centers of these two circles in five-dimensions are displaced by a constant or variable vector $\vec{\ell}(\phi)$ (44) with magnitude $\ell(\phi)$ (45) at each constant or variable phase $\phi$, defined in terms of perpendicular coordinates $\left(\hat{\boldsymbol{u}}_{1}, \hat{\boldsymbol{u}}_{2}, \hat{\boldsymbol{u}}_{3}, \hat{\boldsymbol{u}}_{4}, \hat{\boldsymbol{u}}_{5}\right)$.

$$
\begin{gathered}
\vec{\ell}=\vec{\ell}(\phi)=\hat{\boldsymbol{u}}_{1} \ell_{u_{1}}(\phi)+\hat{\boldsymbol{u}}_{2} \ell_{u_{2}}(\phi)+\hat{\boldsymbol{u}}_{3} \ell_{u_{3}}(\phi)+\hat{\boldsymbol{u}}_{4} \ell_{u_{4}}(\phi)+\hat{\boldsymbol{u}}_{5} \ell_{u_{5}}(\phi) \\
|\vec{\ell}(\phi)|=\ell(\phi)=\sqrt{\ell^{2}(\phi)}=\sqrt{\vec{\ell}(\phi) \cdot \vec{\ell}(\phi)}=\sqrt{\ell_{u_{1}}{ }^{2}(\phi)+\ell_{u_{2}}{ }^{2}(\phi)+\ell_{u_{3}}{ }^{2}(\phi)+\ell_{u_{4}}{ }^{2}(\phi)+\ell_{u_{5}}{ }^{2}(\phi)}
\end{gathered}
$$

Similar to the case in three dimensions for $\overrightarrow{\boldsymbol{d}}(\phi)(11)$, the vector distance between points $\mathbf{P}_{1}$ phased at $\left(\phi+\phi_{0}\right)$ and $\mathbf{P}_{2}$ phased at $(\phi)$ on the two respective circles in five dimensions, at any value of the phase $\phi$, can be equivalently expressed as $\overrightarrow{\boldsymbol{d}}(\phi)$ (46) in terms of virtual vectors $\overrightarrow{\mathbf{X}}(\phi)$ (47) and $\overrightarrow{\mathbf{Y}}(\phi)$ (48), defined utilizing $\overrightarrow{\boldsymbol{r}}_{1}$ (40) - (41) and $\overrightarrow{\boldsymbol{r}}_{2}$ (42).

$$
\begin{aligned}
\overrightarrow{\boldsymbol{d}}(\phi)= & \overrightarrow{\mathbf{X}}(\phi)+\overrightarrow{\mathbf{Y}}(\phi)+\vec{\ell}(\phi) \quad \text { where } \quad \overrightarrow{\boldsymbol{r}_{1}}(\phi)-\overrightarrow{\boldsymbol{r}_{2}}(\phi)=\overrightarrow{\mathbf{X}}(\phi)+\overrightarrow{\mathbf{Y}}(\phi)=\overrightarrow{\boldsymbol{a}} \operatorname{Cos} \phi+\overrightarrow{\boldsymbol{b}} \operatorname{Sin} \phi \\
\overrightarrow{\mathbf{X}}(\phi)= & \overrightarrow{\boldsymbol{a}} \operatorname{Cos} \phi \quad ; \quad \overrightarrow{\mathbf{X}}(\phi) \cdot \overrightarrow{\mathbf{X}}(\phi)=X^{2}(\phi)=\overrightarrow{\boldsymbol{a}} \cdot \overrightarrow{\boldsymbol{a}} \operatorname{Cos}^{2} \phi=a^{2} \operatorname{Cos}^{2} \phi \quad ; \quad|\overrightarrow{\mathbf{X}}(\phi)|=X(\phi) \\
\overrightarrow{\mathbf{Y}}(\phi)= & \overrightarrow{\boldsymbol{b}} \operatorname{Sin} \phi \quad ; \quad \overrightarrow{\mathbf{Y}}(\phi) \cdot \overrightarrow{\mathbf{Y}}(\phi)=Y^{2}(\phi)=\overrightarrow{\boldsymbol{b}} \cdot \overrightarrow{\boldsymbol{b}} \operatorname{Sin}^{2} \phi=b^{2} \operatorname{Sin}^{2} \phi \quad ;|\overrightarrow{\mathbf{Y}}(\phi)|=Y(\phi) \\
\overrightarrow{\boldsymbol{a}}= & \hat{\boldsymbol{u}}_{1}\left(r_{1} \operatorname{Cos} \beta_{1} \operatorname{Cos} \beta_{3} \operatorname{Cos} \phi_{0}-r_{2}\right)+\hat{\boldsymbol{u}}_{2} r_{1} \operatorname{Cos} \beta_{2} \operatorname{Sin} \phi_{0}+\hat{\boldsymbol{u}}_{3} r_{1} \operatorname{Sin} \beta_{1} \operatorname{Cos} \beta_{3} \operatorname{Cos} \phi_{0} \\
& +\hat{\boldsymbol{u}}_{4} r_{1} \operatorname{Sin} \beta_{2} \operatorname{Sin} \phi_{0}+\hat{\boldsymbol{u}}_{5} r_{1} \operatorname{Sin} \beta_{3} \operatorname{Cos} \phi_{0} \Rightarrow \overrightarrow{\boldsymbol{a}}=\left[\overrightarrow{\boldsymbol{r}}_{1}(\phi)-\overrightarrow{\boldsymbol{r}_{2}}(\phi)\right](\phi=0) \\
\overrightarrow{\boldsymbol{b}}= & -\hat{\boldsymbol{u}}_{1} r_{1} \operatorname{Cos} \beta_{1} \operatorname{Cos} \beta_{3} \operatorname{Sin} \phi_{0}+\hat{\boldsymbol{u}}_{2}\left(r_{1} \operatorname{Cos} \beta_{2} \operatorname{Cos} \phi_{0}-r_{2}\right)-\hat{\boldsymbol{u}}_{3} r_{1} \operatorname{Sin} \beta_{1} \operatorname{Cos} \beta_{3} \operatorname{Sin} \phi_{0} \\
& +\hat{\boldsymbol{u}}_{4} r_{1} \operatorname{Sin} \beta_{2} \operatorname{Cos} \phi_{0}-\hat{\boldsymbol{u}}_{5} r_{1} \operatorname{Sin} \beta_{3} \operatorname{Sin} \phi_{0} \Rightarrow \overrightarrow{\boldsymbol{b}}=\left[\overrightarrow{\boldsymbol{r}}_{1}(\phi)-\overrightarrow{\boldsymbol{r}_{2}}(\phi)\right]\left(\phi=\frac{\pi}{2}\right)
\end{aligned}
$$




$$
\begin{gathered}
\overrightarrow{\boldsymbol{a}} \cdot \overrightarrow{\boldsymbol{a}}=a^{2}=r_{1}^{2}-2 r_{1} r_{2} \operatorname{Cos} \beta_{1} \operatorname{Cos} \beta_{3} \operatorname{Cos} \phi_{0}+r_{2}^{2} ;|\overrightarrow{\boldsymbol{a}}|=a \\
\overrightarrow{\boldsymbol{b}} \cdot \overrightarrow{\boldsymbol{b}}=b^{2}=r_{1}^{2}-2 r_{1} r_{2} \operatorname{Cos} \beta_{2} \operatorname{Cos} \phi_{0}+r_{2}^{2} ; \quad|\overrightarrow{\boldsymbol{b}}|=b \\
\overrightarrow{\boldsymbol{a}} \cdot \overrightarrow{\boldsymbol{b}}=r_{1} r_{2}\left(\operatorname{Cos} \beta_{1} \operatorname{Cos} \beta_{3}-\operatorname{Cos} \beta_{2}\right) \operatorname{Sin} \phi_{0}
\end{gathered}
$$

Based on the definition of $\overrightarrow{\mathbf{X}}(\phi)$ (47) from (46) and the definition of $\overrightarrow{\mathbf{Y}}(\phi)$ (48) from (46), the $\overrightarrow{\boldsymbol{a}}$ (49) and $\overrightarrow{\boldsymbol{b}}$ (50) vectors can be defined, which turn out to be the semi-major ${ }^{3}$ and semiminor $^{3}$ axis vectors of the relative ellipse ${ }^{2}$ formed based on the distance $\vec{d}(\phi)$ (46) between points $\mathbf{P}_{1}$ and $\mathbf{P}_{2}$, with magnitudes $a(51)$ and $b$ (52), respectively, and with Dot Product ${ }^{1}$ $(\overrightarrow{\boldsymbol{a}} \cdot \overrightarrow{\boldsymbol{b}})$ (53). According to definitions of vectors $\overrightarrow{\mathbf{X}}(\phi)$ (47), $\overrightarrow{\mathbf{Y}}(\phi)$ (48), $\overrightarrow{\boldsymbol{a}}$ (49), and $\overrightarrow{\boldsymbol{b}}(50)$, as described in (40) - (53), the relation in (19) is valid and holds for all $\phi$. Therefore, the relation in (19) reveals the validity of (20) and (21) for vector pair $[\overrightarrow{\mathbf{X}}(\phi), \overrightarrow{\mathbf{Y}}(\phi)]$ (47) - (48) and their magnitude pair $[X(\phi), Y(\phi)](47)$ - (48), respectively. As (21) is the defining equation of an ellipse ${ }^{2}$ in two dimensions, where $a(51)$ is the semi-major ${ }^{3}$ axis and $b(52)$ is the semi-minor ${ }^{3}$ axis of the ellipse ${ }^{2}$ when $(a>b)^{2,3}$ holds, and vice versa, with (21) reducing to the special case of a circle when $(a=b)^{2,3}$ holds, we can claim that (20) indicates that the vector pair $[\overrightarrow{\mathbf{X}}(\phi), \overrightarrow{\mathbf{Y}}(\phi)](47)$ - (48) defines points on a vector ellipse in five dimensions in the most general case. In other words, the vector distance $\overrightarrow{\boldsymbol{d}}(\phi)$ (46) between two points $\mathbf{P}_{1}$ phased at $\left(\phi+\phi_{0}\right)$ and $\mathbf{P}_{2}$ phased at $(\phi)$ on two respective circles in five dimensions, whose centers are displaced by a constant or variable vector $\vec{\ell}(\phi)$ (44), can equivalently be mathematically expressed and interpreted as the distance of points on a virtual vector ellipse, whose locations with respect to a virtual origin at each $\phi$ are determined by the sum of vector pair $[\overrightarrow{\mathbf{X}}(\phi), \overrightarrow{\mathbf{Y}}(\phi)]$ (47) - (48), from another fixed or moving point displaced from the same virtual origin of the ellipse by a constant or variable vector $[-\vec{\ell}(\phi)](44), \overrightarrow{\boldsymbol{a}}$ (49) and $\overrightarrow{\boldsymbol{b}}$ (50) being the fixed or variable semi-major ${ }^{3}$ and semi-minor ${ }^{3}$ axis vectors of the vector ellipse. This result is mathematically valid even when the phase difference $\phi_{0}$ is a variable function of time $(t)$, i.e. even if $\phi_{0}=\phi_{0}(t)$. This is a finding presented in this Article for two circles in five dimensions. 
Square of the focal ${ }^{2,4}$ distance $\left(c^{2}\right)(54)$ of this vector ellipse ${ }^{2}$ can be found using (51) - (52), and its eccentricity ${ }^{2} e(55)$ can be determined using (51) - (52) and (54).

$$
\begin{aligned}
& c^{2}=\left|a^{2}-b^{2}\right|=2 r_{1} r_{2}\left(\operatorname{Cos} \beta_{2}-\operatorname{Cos} \beta_{1} \operatorname{Cos} \beta_{3}\right)\left|\operatorname{Cos} \phi_{0}\right| \quad \text { (Focal Distance Squared) } \\
& e=\left\{\begin{array}{l}
\frac{c}{a}=\sqrt{\frac{2 r_{1} r_{2}\left(\operatorname{Cos} \beta_{2}-\operatorname{Cos} \beta_{1} \operatorname{Cos} \beta_{3}\right)\left|\operatorname{Cos} \phi_{0}\right|}{r_{1}^{2}-2 r_{1} r_{2} \operatorname{Cos} \beta_{1} \operatorname{Cos} \beta_{3} \operatorname{Cos} \phi_{0}+r_{2}^{2}}} ; \text { if } a>b \\
\frac{c}{b}=\sqrt{\frac{2 r_{1} r_{2}\left(\operatorname{Cos} \beta_{2}-\operatorname{Cos} \beta_{1} \operatorname{Cos} \beta_{3}\right)\left|\operatorname{Cos} \phi_{0}\right|}{r_{1}^{2}-2 r_{1} r_{2} \operatorname{Cos} \beta_{2} \operatorname{Cos} \phi_{0}+r_{2}^{2}}} ; \text { if } a<b
\end{array} \quad\right. \text { (Eccentricity) }
\end{aligned}
$$

Based on the sequence of reasoning and mathematical derivation through (1) - (53), we can reach the general conclusion in (56) - (73) for the case of two circles in multiple dimensions.

For the case of two circles in multiple dimensions, in matrix notation, for one of the circles we can define the vector radius as $\vec{r}_{1}(56)$ in odd $(2 n+1)$ number of dimensions defined in terms of perpendicular coordinates $\left(\hat{\boldsymbol{u}}_{1}, \hat{\boldsymbol{u}}_{2}, \hat{\boldsymbol{u}}_{3}, \hat{\boldsymbol{u}}_{4}, \ldots, \hat{\boldsymbol{u}}_{(2 n)}, \hat{\boldsymbol{u}}_{(2 n+1)}\right)$, or as $\overrightarrow{\boldsymbol{r}}_{1}(57)$ in even $(2 n)$ number of dimensions defined in terms of perpendicular coordinates $\left(\hat{\boldsymbol{u}}_{1}, \hat{\boldsymbol{u}}_{2}, \hat{\boldsymbol{u}}_{3}, \hat{\boldsymbol{u}}_{4}, \ldots, \hat{\boldsymbol{u}}_{(2 n-1)}, \hat{\boldsymbol{u}}_{(2 n)}\right),(n \geq 1)$ being a positive integer, and we can define the vector radius $\vec{r}_{2}$ (58) for the other of the two circles, in the simplest case, with constant magnitudes $r_{1}$ (59) and $r_{2}$ (59), respectively, which are the radius vectors at point $\mathbf{P}_{1}$ phased at $\left(\phi+\phi_{0}\right)$ and $\mathbf{P}_{2}$ phased at $(\phi)$ on the two circles, respectively, that are phased apart by a constant or time $(t)$-dependent angle $\phi_{0}(56)$ - (57).

Note that $\beta_{1}(56)-(57)$ is taken to be the constant inclination angle between these two circles in the $\hat{\boldsymbol{u}}_{1}-\hat{\boldsymbol{u}}_{3}$ dimension plane, $\beta_{2}(56)-(57)$ is taken to be the constant inclination angle between these two circles in the $\hat{\boldsymbol{u}}_{2}-\hat{\boldsymbol{u}}_{4}$ dimension plane, $\beta_{3}(56)-(57)$ is taken to be the constant inclination angle of these two circles between the $\hat{\boldsymbol{u}}_{1}-\hat{\boldsymbol{u}}_{3}$ dimension plane and the $\hat{\boldsymbol{u}}_{5}$ dimension, $\beta_{4}(56)-(57)$ is taken to be the constant inclination angle of these two circles between $\hat{\boldsymbol{u}}_{2}-\hat{\boldsymbol{u}}_{4}$ dimension plane and the $\hat{\boldsymbol{u}}_{6}$ dimension, $\beta_{(2 n-1)}(56)-(57)$ is taken to be the constant inclination angle of these two circles between the $\hat{\boldsymbol{u}}_{1}-\hat{\boldsymbol{u}}_{3}-\ldots-\hat{\boldsymbol{u}}_{(2 n-3)}-\hat{\boldsymbol{u}}_{(2 n-1)}$ dimension complex and the $\hat{\boldsymbol{u}}_{(2 n+1)}$ dimension, and $\beta_{(2 n-2)}(56)-(57)$ is taken to be the constant 
inclination angle of these two circles between the $\hat{\boldsymbol{u}}_{2}-\hat{\boldsymbol{u}}_{4}-\ldots-\hat{\boldsymbol{u}}_{(2 n-4)}-\hat{\boldsymbol{u}}_{(2 n-2)}$ dimension complex and the $\hat{\boldsymbol{u}}_{(2 n)}$ dimension.

$$
\overrightarrow{\boldsymbol{r}}_{1}=\overrightarrow{\boldsymbol{r}}_{1}\left(\phi+\phi_{0}\right)=r_{1}\left[\begin{array}{c}
\hat{\boldsymbol{u}}_{1} \operatorname{Cos}\left(\phi+\phi_{0}\right) \\
\hat{\boldsymbol{u}}_{2} \operatorname{Sin}\left(\phi+\phi_{0}\right) \\
\hat{\boldsymbol{u}}_{3} \operatorname{Cos}\left(\phi+\phi_{0}\right) \\
\hat{\boldsymbol{u}}_{4} \operatorname{Sin}\left(\phi+\phi_{0}\right) \\
\hat{\boldsymbol{u}}_{5} \operatorname{Cos}\left(\phi+\phi_{0}\right) \\
\hat{\boldsymbol{u}}_{6} \operatorname{Sin}\left(\phi+\phi_{0}\right) \\
\hat{\boldsymbol{u}}_{7} \operatorname{Cos}\left(\phi+\phi_{0}\right) \\
\vdots \\
\hat{\boldsymbol{u}}_{(2 n-2)} \operatorname{Sin}\left(\phi+\phi_{0}\right) \\
\hat{\boldsymbol{u}}_{(2 n-1)} \operatorname{Cos}\left(\phi+\phi_{0}\right) \\
\hat{\boldsymbol{u}}_{(2 n)} \operatorname{Sin}\left(\phi+\phi_{0}\right) \\
\hat{\boldsymbol{u}}_{(2 n+1)} \operatorname{Cos}\left(\phi+\phi_{0}\right)
\end{array}\right]_{1 \times(2 n+1)}\left[\begin{array}{c}
\operatorname{Cos} \beta_{1} \operatorname{Cos} \beta_{3} \operatorname{Cos} \beta_{5} \ldots \operatorname{Cos} \beta_{(2 n-1)} \\
\operatorname{Cos} \beta_{2} \operatorname{Cos} \beta_{4} \ldots \operatorname{Cos} \beta_{(2 n-2)} \\
\operatorname{Sin} \beta_{1} \operatorname{Cos} \beta_{3} \operatorname{Cos} \beta_{5} \ldots \operatorname{Cos} \beta_{(2 n-1)} \\
\operatorname{Sin} \beta_{2} \operatorname{Cos} \beta_{4} \ldots \operatorname{Cos} \beta_{(2 n-2)} \\
\operatorname{Sin} \beta_{3} \operatorname{Cos} \beta_{5} \ldots \operatorname{Cos} \beta_{(2 n-1)} \\
\operatorname{Sin} \beta_{4} \operatorname{Cos} \beta_{6} \ldots \operatorname{Cos} \beta_{(2 n-2)} \\
\operatorname{Sin} \beta_{5} \operatorname{Cos} \beta_{7} \ldots \operatorname{Cos} \beta_{(2 n-1)} \\
\vdots \\
\operatorname{Sin} \beta_{(2 n-4)} \operatorname{Cos} \beta_{(2 n-2)} \\
\operatorname{Sin} \beta_{(2 n-3)} \operatorname{Cos} \beta_{(2 n-1)} \\
\operatorname{Sin} \beta_{(2 n-2)} \\
\operatorname{Sin} \beta_{(2 n-1)}
\end{array}\right]_{(2 n+1) \times 1}\left[\begin{array}{c}
(2 n+1) \\
\text { odd } \\
\text { number } \\
\text { of } \\
\text { dim.sions } \\
n \geq 1 \\
\text { integer } \\
\text { Only } \\
\beta_{i} \\
\text { terms } \\
\text { with } \\
i>0
\end{array}\right.
$$

$$
\begin{aligned}
& \overrightarrow{\boldsymbol{r}}_{1}=\overrightarrow{\boldsymbol{r}}_{1}\left(\phi+\phi_{0}\right)=r_{1}\left[\begin{array}{c}
\hat{\boldsymbol{u}}_{1} \operatorname{Cos}\left(\phi+\phi_{0}\right) \\
\hat{\boldsymbol{u}}_{2} \operatorname{Sin}\left(\phi+\phi_{0}\right) \\
\hat{\boldsymbol{u}}_{3} \operatorname{Cos}\left(\phi+\phi_{0}\right) \\
\hat{\boldsymbol{u}}_{4} \operatorname{Sin}\left(\phi+\phi_{0}\right) \\
\hat{\boldsymbol{u}}_{5} \operatorname{Cos}\left(\phi+\phi_{0}\right) \\
\hat{\boldsymbol{u}}_{6} \operatorname{Sin}\left(\phi+\phi_{0}\right) \\
\vdots \\
\hat{\boldsymbol{u}}_{(2 n-3)} \operatorname{Cos}\left(\phi+\phi_{0}\right) \\
\hat{\boldsymbol{u}}_{(2 n-2)} \operatorname{Sin}\left(\phi+\phi_{0}\right) \\
\hat{\boldsymbol{u}}_{(2 n-1)} \operatorname{Cos}\left(\phi+\phi_{0}\right) \\
\hat{\boldsymbol{u}}_{(2 n)} \operatorname{Sin}\left(\phi+\phi_{0}\right)
\end{array}\right]_{1 \times(2 n)}\left[\begin{array}{c}
\operatorname{Cos} \beta_{1} \operatorname{Cos} \beta_{3} \operatorname{Cos} \beta_{5} \ldots \operatorname{Cos} \beta_{(2 n-3)} \\
\operatorname{Cos} \beta_{2} \operatorname{Cos} \beta_{4} \ldots \operatorname{Cos} \beta_{(2 n-2)} \\
\operatorname{Sin} \beta_{1} \operatorname{Cos} \beta_{3} \operatorname{Cos} \beta_{5} \ldots \operatorname{Cos} \beta_{(2 n-3)} \\
\operatorname{Sin} \beta_{2} \operatorname{Cos} \beta_{4} \ldots \operatorname{Cos} \beta_{(2 n-2)} \\
\operatorname{Sin} \beta_{3} \operatorname{Cos} \beta_{5} \ldots \operatorname{Cos} \beta_{(2 n-3)} \\
\operatorname{Sin} \beta_{4} \operatorname{Cos} \beta_{6} \ldots \operatorname{Cos} \beta_{(2 n-2)} \\
\vdots \\
\operatorname{Sin} \beta_{(2 n-5)} \operatorname{Cos} \beta_{(2 n-3)} \\
\operatorname{Sin} \beta_{(2 n-4)} \operatorname{Cos} \beta_{(2 n-2)} \\
\operatorname{Sin} \beta_{(2 n-3)} \\
\operatorname{Sin} \beta_{(2 n-2)}
\end{array}\right]_{(2 n) \times 1}\left[\begin{array}{c}
(2 n) \text { even } \\
\text { number } \\
\text { of } \\
\text { dim.sions } \\
\text { integer } \\
\text { Only } \\
\beta_{i} \\
\text { terms } \\
\text { with }
\end{array}\right. \\
& \overrightarrow{\boldsymbol{r}}_{2}=\overrightarrow{\boldsymbol{r}}_{2}(\phi)=\hat{\boldsymbol{u}}_{1} r_{2} \operatorname{Cos} \phi+\hat{\boldsymbol{u}}_{2} r_{2} \operatorname{Sin} \phi \\
& \overrightarrow{\boldsymbol{r}}_{1} \cdot \overrightarrow{\boldsymbol{r}}_{1}=r_{1}^{2} \quad ; \quad\left|\overrightarrow{\boldsymbol{r}}_{1}\right|=r_{1}=\sqrt{\overrightarrow{\boldsymbol{r}}_{1} \cdot \overrightarrow{\boldsymbol{r}}_{1}} \quad ; \quad \overrightarrow{\boldsymbol{r}}_{2} \cdot \overrightarrow{\boldsymbol{r}}_{2}=r_{2}^{2} \quad ; \quad\left|\overrightarrow{\boldsymbol{r}}_{2}\right|=r_{2}=\sqrt{\overrightarrow{\boldsymbol{r}}_{2} \cdot \overrightarrow{\boldsymbol{r}}_{2}}
\end{aligned}
$$


In odd $(2 n+1)$ number of dimensions, $\vec{r}_{1}(56)$ can be restated as $\vec{r}_{1}(60)$ in terms of $\operatorname{Cos} \phi$ and $\operatorname{Sin} \phi$ terms. In even $(2 n)$ number of dimensions, $\overrightarrow{\boldsymbol{r}}_{1}(57)$ can be restated as $\overrightarrow{\boldsymbol{r}}_{1}(61)$ in terms of $\operatorname{Cos} \phi$ and $\operatorname{Sin} \phi$ terms.

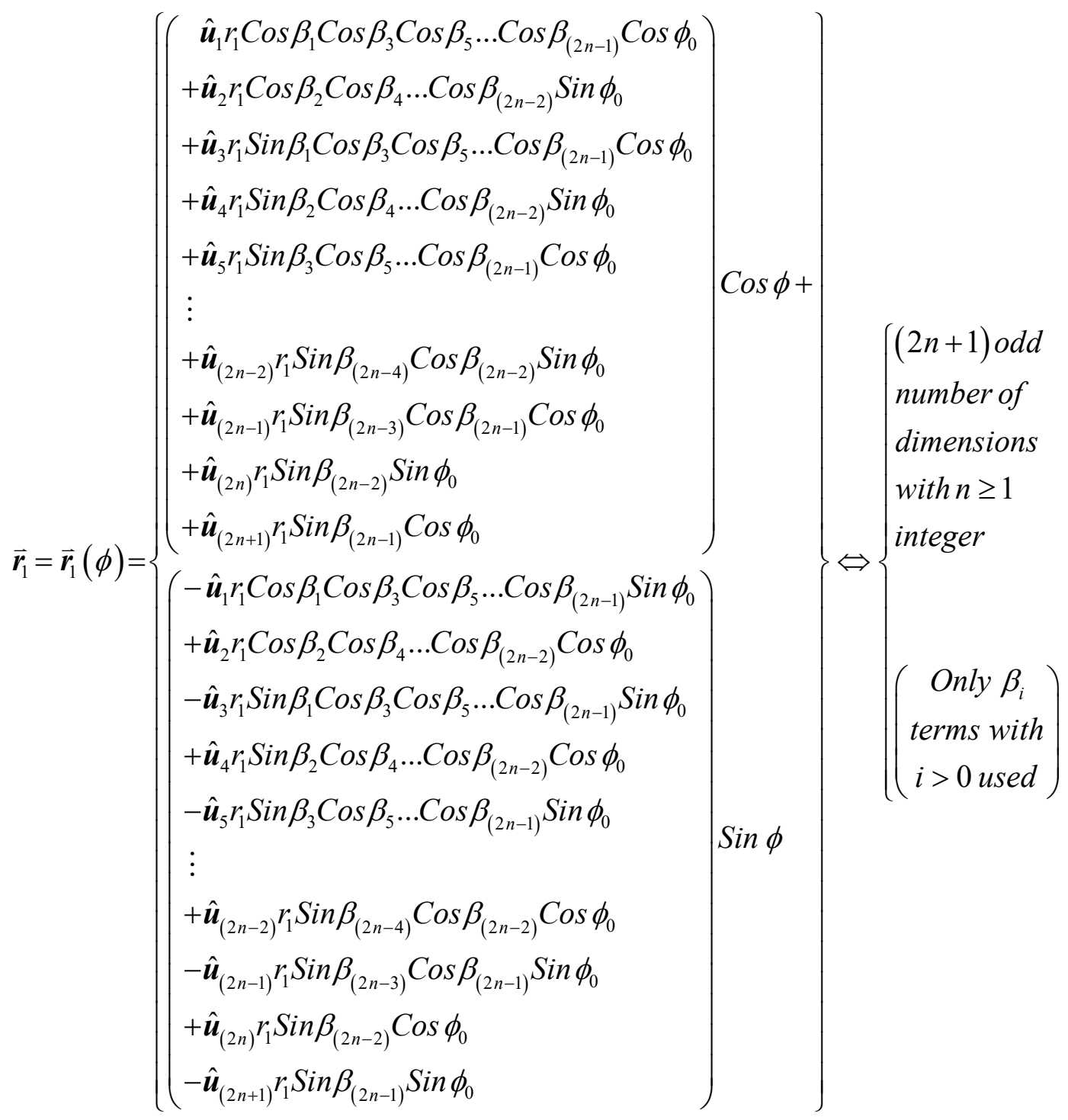




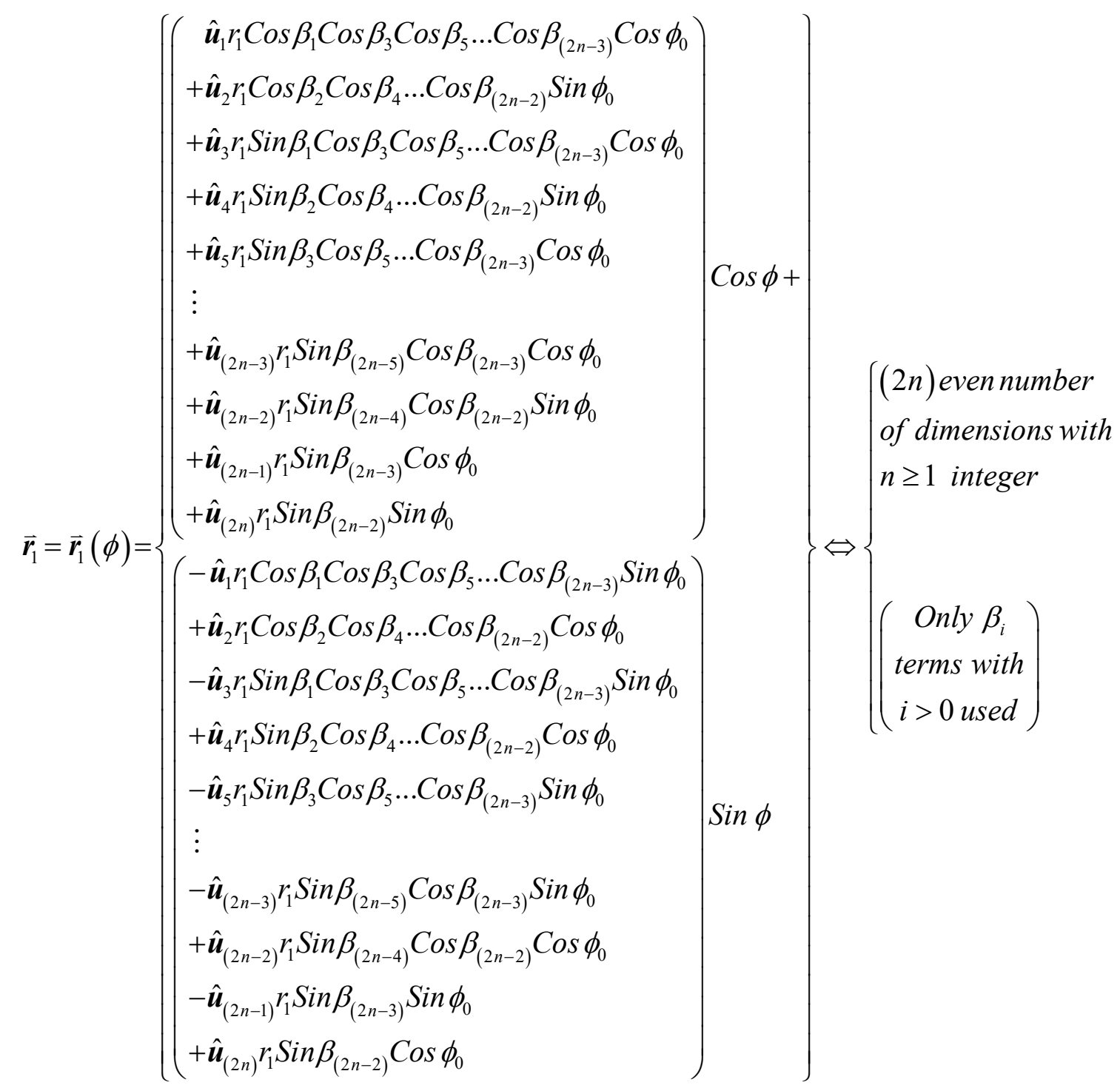

Centers of these two circles in multiple dimensions are displaced by a constant or variable vector $\vec{\ell}(\phi)$ (62) with magnitude $\ell(\phi)$ (63) at each constant or variable phase $\phi$, defined in terms of perpendicular coordinates $\left(\hat{\boldsymbol{u}}_{1}, \hat{\boldsymbol{u}}_{2}, \hat{\boldsymbol{u}}_{3}, \hat{\boldsymbol{u}}_{4}, \ldots, \hat{\boldsymbol{u}}_{(2 n)}, \hat{\boldsymbol{u}}_{(2 n+1)}\right)$ in odd $(2 n+1)$ number of dimensions, or defined in terms of perpendicular coordinates $\left(\hat{\boldsymbol{u}}_{1}, \hat{\boldsymbol{u}}_{2}, \hat{\boldsymbol{u}}_{3}, \hat{\boldsymbol{u}}_{4}, \ldots, \hat{\boldsymbol{u}}_{(2 n-1)}, \hat{\boldsymbol{u}}_{(2 n)}\right)$ in even $(2 n)$ number of dimensions, $(n \geq 1)$ being a positive integer. $\vec{\ell}=\vec{\ell}(\phi)=\left\{\begin{array}{l}\hat{\boldsymbol{u}}_{1} \ell_{u_{1}}(\phi)+\hat{\boldsymbol{u}}_{2} \ell_{u_{2}}(\phi)+\ldots+\hat{\boldsymbol{u}}_{(2 n)} \ell_{u_{(2 n)}}(\phi)+\hat{\boldsymbol{u}}_{(2 n+1)} \ell_{u_{(2 n+1)}}(\phi), \text { odd \# of dim.sions } \\ \hat{\boldsymbol{u}}_{1} \ell_{u_{1}}(\phi)+\hat{\boldsymbol{u}}_{2} \ell_{u_{2}}(\phi)+\ldots+\hat{\boldsymbol{u}}_{(2 n-1)} \ell_{u_{(2 n-1)}}(\phi)+\hat{\boldsymbol{u}}_{(2 n)} \ell_{u_{(2 n)}}(\phi), \text { even \# of dim.sions }\end{array}\right.$
$\ell^{2}(\phi)=\vec{\ell}(\phi) \cdot \vec{\ell}(\phi)=\left\{\begin{array}{l}\ell_{u_{1}}^{2}(\phi)+\ell_{u_{2}}^{2}(\phi)+\ldots+\ell_{u_{(2 n)}}^{2}(\phi)+\ell_{u_{(2 n+1)}}^{2}(\phi), \text { odd \# of dim.sions } \\ \ell_{u_{1}}^{2}(\phi)+\ell_{u_{2}}^{2}(\phi)+\ldots+\ell_{u_{(2 n-1)}}^{2}(\phi)+\ell_{u_{(2 n)}}^{2}(\phi), \text { even \# of dim.sions }\end{array}\right.$ 
Similar to the case in three dimensions for $\overrightarrow{\boldsymbol{d}}(\phi)(11)$, we see that the vector distance between points $\mathbf{P}_{1}$ phased at $\left(\phi+\phi_{0}\right)$ and $\mathbf{P}_{2}$ phased at $(\phi)$ on the two respective circles in any number of multiple dimensions, at any value of the phase $\phi$, can be equivalently expressed as $\overrightarrow{\boldsymbol{d}}(\phi)$ (64) in terms of virtual vectors $\overrightarrow{\mathbf{X}}(\phi)$ (65) and $\overrightarrow{\mathbf{Y}}(\phi)$ (66), defined utilizing $\overrightarrow{\boldsymbol{r}}_{1}(60)-(61)$ and $\overrightarrow{\boldsymbol{r}}_{2}(58)$.

$$
\begin{aligned}
& \overrightarrow{\boldsymbol{d}}(\phi)=\overrightarrow{\mathbf{X}}(\phi)+\overrightarrow{\mathbf{Y}}(\phi)+\vec{\ell}(\phi) \quad \text { where } \quad \overrightarrow{\boldsymbol{r}_{1}}(\phi)-\overrightarrow{\boldsymbol{r}_{2}}(\phi)=\overrightarrow{\mathbf{X}}(\phi)+\overrightarrow{\mathbf{Y}}(\phi)=\overrightarrow{\boldsymbol{a}} \operatorname{Cos} \phi+\overrightarrow{\boldsymbol{b}} \operatorname{Sin} \phi \\
& \overrightarrow{\mathbf{X}}(\phi)=\overrightarrow{\boldsymbol{a}} \operatorname{Cos} \phi \quad ; \quad \overrightarrow{\mathbf{X}}(\phi) \cdot \overrightarrow{\mathbf{X}}(\phi)=X^{2}(\phi)=\overrightarrow{\boldsymbol{a}} \cdot \overrightarrow{\boldsymbol{a}} \operatorname{Cos}^{2} \phi=a^{2} \operatorname{Cos}^{2} \phi \quad ; \quad|\overrightarrow{\mathbf{X}}(\phi)|=X(\phi) \\
& \overrightarrow{\mathbf{Y}}(\phi)=\overrightarrow{\boldsymbol{b}} \operatorname{Sin} \phi \quad ; \quad \overrightarrow{\mathbf{Y}}(\phi) \cdot \overrightarrow{\mathbf{Y}}(\phi)=Y^{2}(\phi)=\overrightarrow{\boldsymbol{b}} \cdot \overrightarrow{\boldsymbol{b}} \operatorname{Sin}^{2} \phi=b^{2} \operatorname{Sin}^{2} \phi \quad ; \quad|\overrightarrow{\mathbf{Y}}(\phi)|=Y(\phi)
\end{aligned}
$$

Based on the definition of $\overrightarrow{\mathbf{X}}(\phi)$ (65) from (64) and the definition of $\overrightarrow{\mathbf{Y}}(\phi)$ (66) from (64), $\overrightarrow{\boldsymbol{a}}(67)$ - (68) and $\overrightarrow{\boldsymbol{b}}(70)$ - (71) vectors can be defined, which turn out to be the semi-major ${ }^{3}$ and semi-minor ${ }^{3}$ axis vectors of the relative ellipse ${ }^{2}$ formed based on the distance $\overrightarrow{\boldsymbol{d}}(\phi)(64)$ between points $\mathbf{P}_{1}$ and $\mathbf{P}_{2}$, with magnitudes $a$ (69) and $b$ (72), respectively, and having the $\operatorname{Dot}_{\text {Product }^{1}}(\overrightarrow{\boldsymbol{a}} \cdot \overrightarrow{\boldsymbol{b}})(73)$. According to the definitions of the vectors $\overrightarrow{\mathbf{X}}(\phi)(65), \overrightarrow{\mathbf{Y}}(\phi)(66)$, $\overrightarrow{\boldsymbol{a}}$ (67) - (68), and $\overrightarrow{\boldsymbol{b}}$ (70) - (71), as described in (56) - (73), the relation in (19) is valid and holds for all $\phi$. Therefore, the relation in (19) reveals the validity of (20) and (21) for the vector pair $[\overrightarrow{\mathbf{X}}(\phi), \overrightarrow{\mathbf{Y}}(\phi)](65)$ - (66) and their magnitude pair $[X(\phi), Y(\phi)](65)$ - (66), respectively. As (21) is the defining equation of an ellipse ${ }^{2}$ in two dimensions, where $a$ (69) is the semi-major ${ }^{3}$ axis and $b(72)$ is the semi-minor ${ }^{3}$ axis of the ellipse $e^{2}$ when $(a>b)^{2,3}$ holds, and vice versa, with (21) reducing to the special case of a circle when $(a=b)^{2,3}$ holds, we can claim that (20) indicates that the vector pair $[\overrightarrow{\mathbf{X}}(\phi), \overrightarrow{\mathbf{Y}}(\phi)](65)$ - (66) defines points on a vector ellipse in multiple number of dimensions in the most general case. 


$$
\overrightarrow{\boldsymbol{a}}=\left[\overrightarrow{\boldsymbol{r}_{1}}(\phi)-\overrightarrow{\boldsymbol{r}}_{2}(\phi)\right](\phi=0)=\left(\begin{array}{l}
\hat{\boldsymbol{u}}_{1}\left(r_{1} \operatorname{Cos} \beta_{1} \operatorname{Cos} \beta_{3} \operatorname{Cos} \beta_{5} \ldots \operatorname{Cos} \beta_{(2 n-1)} \operatorname{Cos} \phi_{0}-r_{2}\right) \\
+\hat{\boldsymbol{u}}_{2} r_{1} \operatorname{Cos} \beta_{2} \operatorname{Cos} \beta_{4} \ldots \operatorname{Cos} \beta_{(2 n-2)} \operatorname{Sin} \phi_{0} \\
+\hat{\boldsymbol{u}}_{3} r_{1} \operatorname{Sin} \beta_{1} \operatorname{Cos} \beta_{3} \operatorname{Cos} \beta_{5} \ldots \operatorname{Cos} \beta_{(2 n-1)} \operatorname{Cos} \phi_{0} \\
+\hat{\boldsymbol{u}}_{4} r_{1} \operatorname{Sin} \beta_{2} \operatorname{Cos} \beta_{4} \ldots \operatorname{Cos} \beta_{(2 n-2)} \operatorname{Sin} \phi_{0} \\
+\hat{\boldsymbol{u}}_{5} r_{1} \operatorname{Sin} \beta_{3} \operatorname{Cos} \beta_{5} \ldots \operatorname{Cos} \beta_{(2 n-1)} \operatorname{Cos} \phi_{0} \\
\vdots \\
+\hat{\boldsymbol{u}}_{(2 n-2)} r_{1} \operatorname{Sin} \beta_{(2 n-4)} \operatorname{Cos} \beta_{(2 n-2)} \operatorname{Sin} \phi_{0} \\
+\hat{\boldsymbol{u}}_{(2 n-1)} r_{1} \operatorname{Sin} \beta_{(2 n-3)} \operatorname{Cos} \beta_{(2 n-1)} \operatorname{Cos} \phi_{0} \\
+\hat{\boldsymbol{u}}_{(2 n)} r_{1} \operatorname{Sin} \beta_{(2 n-2)} \operatorname{Sin} \phi_{0} \\
+\hat{\boldsymbol{u}}_{(2 n+1)} r_{1} \operatorname{Sin} \beta_{(2 n-1)} \operatorname{Cos} \phi_{0} \\
\text { odd } \\
\text { dim.sions } \\
n \geq 1 \\
\text { integer } \\
\text { Only } \\
\beta_{i} \\
\text { terms } \\
\text { with } \\
i>0
\end{array}\right.
$$

$$
\overrightarrow{\boldsymbol{a}}=\left[\overrightarrow{\boldsymbol{r}_{1}}(\phi)-\overrightarrow{\boldsymbol{r}_{2}}(\phi)\right](\phi=0)=\left(\begin{array}{l}
\hat{\boldsymbol{u}}_{1}\left(r_{1} \operatorname{Cos} \beta_{1} \operatorname{Cos} \beta_{3} \operatorname{Cos} \beta_{5} \ldots \operatorname{Cos} \beta_{(2 n-3)} \operatorname{Cos} \phi_{0}-r_{2}\right) \\
+\hat{\boldsymbol{u}}_{2} r_{1} \operatorname{Cos} \beta_{2} \operatorname{Cos} \beta_{4} \ldots \operatorname{Cos} \beta_{(2 n-2)} \operatorname{Sin} \phi_{0} \\
+\hat{\boldsymbol{u}}_{3} r_{1} \operatorname{Sin} \beta_{1} \operatorname{Cos} \beta_{3} \operatorname{Cos} \beta_{5} \ldots \operatorname{Cos} \beta_{(2 n-3)} \operatorname{Cos} \phi_{0} \\
+\hat{\boldsymbol{u}}_{4} r_{1} \operatorname{Sin} \beta_{2} \operatorname{Cos} \beta_{4} \ldots \operatorname{Cos} \beta_{(2 n-2)} \operatorname{Sin} \phi_{0} \\
+\hat{\boldsymbol{u}}_{5} r_{1} \operatorname{Sin} \beta_{3} \operatorname{Cos} \beta_{5} \ldots \operatorname{Cos} \beta_{(2 n-3)} \operatorname{Cos} \phi_{0} \\
\vdots \\
+\hat{\boldsymbol{u}}_{(2 n-3)} r_{1} \operatorname{Sin} \beta_{(2 n-5)} \operatorname{Cos} \beta_{(2 n-3)} \operatorname{Cos} \phi_{0} \\
+\hat{\boldsymbol{u}}_{(2 n-2)} r_{1} \operatorname{Sin} \beta_{(2 n-4)} \operatorname{Cos} \beta_{(2 n-2)} \operatorname{Sin} \phi_{0} \\
+\hat{\boldsymbol{u}}_{(2 n-1)} r_{1} \operatorname{Sin} \beta_{(2 n-3)} \operatorname{Cos} \phi_{0} \\
+\hat{\boldsymbol{u}}_{(2 n)} r_{1} \operatorname{Sin} \beta_{(2 n-2)} \operatorname{Sin} \phi_{0} \\
\text { even } \\
\text { dim.sions } \\
n \geq 1 \\
\text { integer } \\
\text { Only } \\
\beta_{i} \\
\text { terms } \\
\text { with } \\
i>0
\end{array}\right.
$$

$\overrightarrow{\boldsymbol{a}} \cdot \overrightarrow{\boldsymbol{a}}=a^{2}=\left\{\begin{array}{l}r_{1}^{2}-2 r_{1} r_{2} \operatorname{Cos} \beta_{1} \operatorname{Cos} \beta_{3} \ldots \operatorname{Cos} \beta_{(2 n-1)} \operatorname{Cos} \phi_{0}+r_{2}^{2}, \text { odd number of dimensions } \\ r_{1}^{2}-2 r_{1} r_{2} \operatorname{Cos} \beta_{1} \operatorname{Cos} \beta_{3} \ldots \operatorname{Cos} \beta_{(2 n-3)} \operatorname{Cos} \phi_{0}+r_{2}^{2}, \text { even number of dimensions }\end{array} ;|\overrightarrow{\boldsymbol{a}}|=a(69)\right.$ 


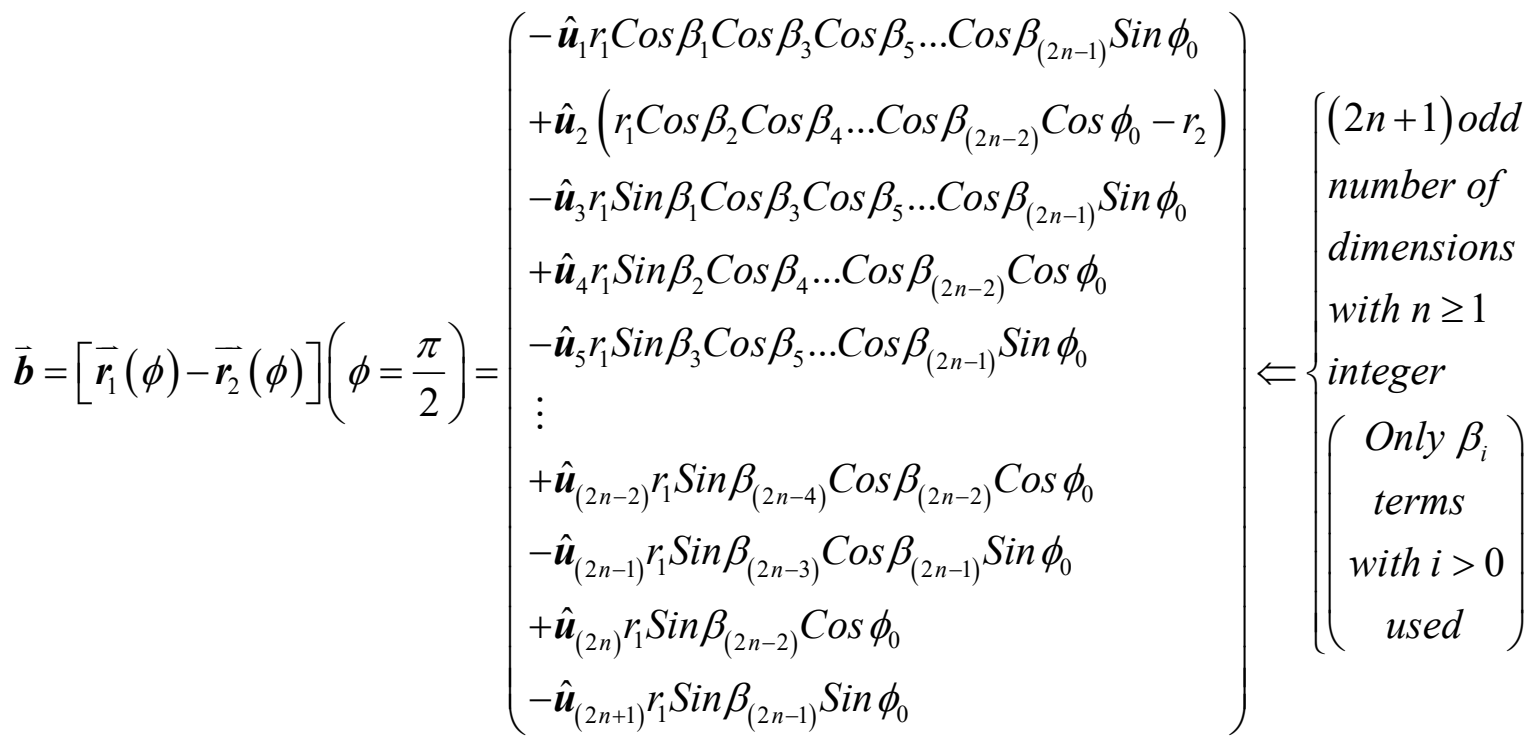

$$
\begin{aligned}
& \overrightarrow{\boldsymbol{b}}=\left[\overrightarrow{\boldsymbol{r}_{1}}(\phi)-\overrightarrow{\boldsymbol{r}_{2}}(\phi)\right]\left(\phi=\frac{\pi}{2}\right)=\left(\begin{array}{l}
-\hat{\boldsymbol{u}}_{1} r_{1} \operatorname{Cos} \beta_{1} \operatorname{Cos} \beta_{3} \operatorname{Cos} \beta_{5} \ldots \operatorname{Cos} \beta_{(2 n-3)} \operatorname{Sin} \phi_{0} \\
+\hat{\boldsymbol{u}}_{2}\left(r_{1} \operatorname{Cos} \beta_{2} \operatorname{Cos} \beta_{4} \ldots \operatorname{Cos} \beta_{(2 n-2)} \operatorname{Cos} \phi_{0}-r_{2}\right) \\
-\hat{\boldsymbol{u}}_{3} r_{1} \operatorname{Sin} \beta_{1} \operatorname{Cos} \beta_{3} \operatorname{Cos} \beta_{5} \ldots \operatorname{Cos} \beta_{(2 n-3)} \operatorname{Sin} \phi_{0} \\
+\hat{\boldsymbol{u}}_{4} r_{1} \operatorname{Sin} \beta_{2} \operatorname{Cos} \beta_{4} \ldots \operatorname{Cos} \beta_{(2 n-2)} \operatorname{Cos} \phi_{0} \\
-\hat{\boldsymbol{u}}_{5} r_{1} \operatorname{Sin} \beta_{3} \operatorname{Cos} \beta_{5} \ldots \operatorname{Cos} \beta_{(2 n-3)} \operatorname{Sin} \phi_{0} \\
\vdots \\
-\hat{\boldsymbol{u}}_{(2 n-3)} r_{1} \operatorname{Sin} \beta_{(2 n-5)} \operatorname{Cos} \beta_{(2 n-3)} \operatorname{Sin} \phi_{0} \\
+\hat{\boldsymbol{u}}_{(2 n-2)} r_{1} \operatorname{Sin} \beta_{(2 n-4)} \operatorname{Cos} \beta_{(2 n-2)} \operatorname{Cos} \phi_{0} \\
-\hat{\boldsymbol{u}}_{(2 n-1)} r_{1} \operatorname{Sin} \beta_{(2 n-3)} \operatorname{Sin} \phi_{0} \\
+\hat{\boldsymbol{u}}_{(2 n)} r_{1} \operatorname{Sin} \beta_{(2 n-2)} \operatorname{Cos} \phi_{0}
\end{array}\right) \Leftrightarrow\left\{\begin{array}{l}
(2 n) \text { even } \\
\text { number of } \\
\text { dimensions } \\
\text { with n } \geq 1 \\
\text { integer } \\
\text { Only } \beta_{i} \\
\text { terms } \\
\text { with i }>0 \\
\text { used }
\end{array}\right)
\end{aligned}
$$

$\overrightarrow{\boldsymbol{b}} \cdot \overrightarrow{\boldsymbol{b}}=b^{2}=r_{1}^{2}-2 r_{1} r_{2} \operatorname{Cos} \beta_{2} \operatorname{Cos} \beta_{4} \ldots \operatorname{Cos} \beta_{(2 n-2)} \operatorname{Cos} \phi_{0}+r_{2}^{2} \quad$ (multiple dimensions) $; \quad|\overrightarrow{\boldsymbol{b}}|=b$

$\overrightarrow{\boldsymbol{a}} \cdot \overrightarrow{\boldsymbol{b}}= \begin{cases}r_{1} r_{2}\left(\left[\operatorname{Cos} \beta_{1} \operatorname{Cos} \beta_{3} \ldots \operatorname{Cos} \beta_{(2 n-1)}\right]-\left[\operatorname{Cos} \beta_{2} \operatorname{Cos} \beta_{4} \ldots \operatorname{Cos} \beta_{(2 n-2)}\right]\right) \operatorname{Sin} \phi_{0} & \begin{array}{l}\text { odd number } \\ \text { of dimensions }\end{array} \\ r_{1} r_{2}\left(\left[\operatorname{Cos} \beta_{1} \operatorname{Cos} \beta_{3} \ldots \operatorname{Cos} \beta_{(2 n-3)}\right]-\left[\operatorname{Cos} \beta_{2} \operatorname{Cos} \beta_{4} \ldots \operatorname{Cos} \beta_{(2 n-2)}\right]\right) \operatorname{Sin} \phi_{0}\left\langle\begin{array}{l}\text { even number } \\ \text { of dimensions }\end{array}\right.\end{cases}$

In other words, the vector distance $\overrightarrow{\boldsymbol{d}}(\phi)$ (64) between two points $\mathbf{P}_{1}$ phased at $\left(\phi+\phi_{0}\right)$ and $\mathbf{P}_{2}$ phased at $(\phi)$ on two respective circles in multiple number of dimensions, whose centers are displaced by a constant or variable vector $\vec{\ell}(\phi)$ (62), can equivalently be mathematically expressed and interpreted as the distance of points on a virtual vector ellipse, whose locations with respect to a virtual origin at each $\phi$ are determined by the sum of vector pair 
$[\overrightarrow{\mathbf{X}}(\phi), \overrightarrow{\mathbf{Y}}(\phi)](65)$ - (66), from another fixed or moving point displaced from the same virtual origin of the ellipse by a constant or variable vector $[-\vec{\ell}(\phi)](62), \overrightarrow{\boldsymbol{a}}(67)$ - (68) and $\overrightarrow{\boldsymbol{b}}$ (70) - (71) vectors being the fixed or variable semi-major ${ }^{3}$ and semi-minor ${ }^{3}$ axis vectors of the vector ellipse. This result is mathematically valid even when the phase difference $\phi_{0}$ is a variable function of time $(t)$, i.e. even if $\phi_{0}=\phi_{0}(t)$. This is the main significant finding which we had aimed to obtain in this Article for two circles in multiple number of dimensions.

Square of the focal ${ }^{2,4}$ distance $\left(c^{2}\right)(74)$ of this vector ellipse ${ }^{2}$ can be found using (69) and (72), and its eccentricity ${ }^{2} e(75)$ can be determined using (69), (72) and (74).

$$
\begin{aligned}
& c^{2}=\left|a^{2}-b^{2}\right| \\
& =\left\{\begin{array}{l}
2 r_{1} r_{2}\left(\operatorname{Cos} \beta_{2} \operatorname{Cos} \beta_{4} \ldots \operatorname{Cos} \beta_{(2 n-2)}-\operatorname{Cos} \beta_{1} \operatorname{Cos} \beta_{3} \ldots \operatorname{Cos} \beta_{(2 n-1)}\right)\left|\operatorname{Cos} \phi_{0}\right|,\left|\begin{array}{l}
\text { odd number } \\
\text { of dimensions }
\end{array}\right\rangle \\
2 r_{1} r_{2}\left(\operatorname{Cos} \beta_{2} \operatorname{Cos} \beta_{4} \ldots \operatorname{Cos} \beta_{(2 n-2)}-\operatorname{Cos} \beta_{1} \operatorname{Cos} \beta_{3} \ldots \operatorname{Cos} \beta_{(2 n-3)}\right)\left|\operatorname{Cos} \phi_{0}\right|,\left\langle\begin{array}{l}
\text { even number } \\
\text { of dimensions }
\end{array}\right\rangle
\end{array}\right.
\end{aligned}
$$

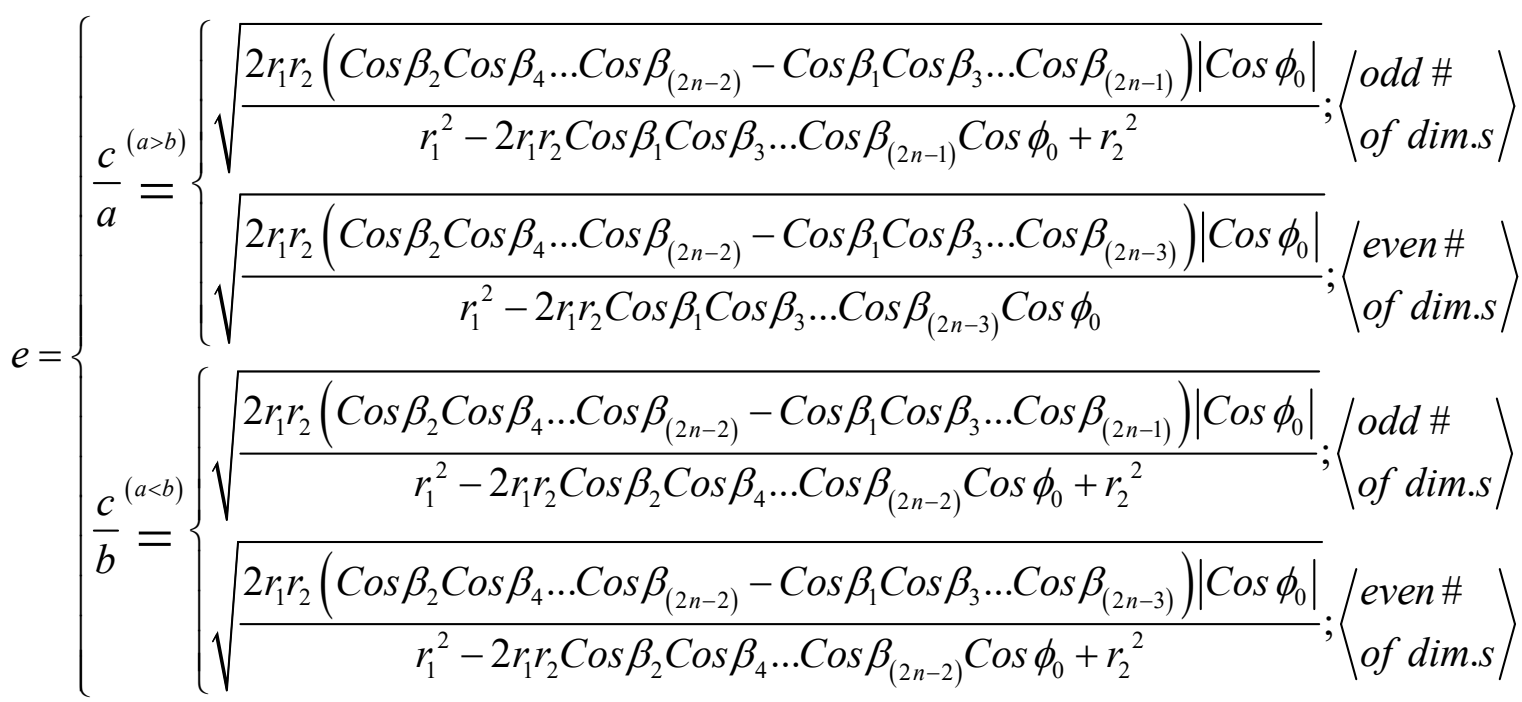

The vector distance $\overrightarrow{\boldsymbol{d}}(\phi)$ (64) at any value of the phase $\phi$, between points $\mathbf{P}_{1}$ phased at $\left(\phi+\phi_{0}\right)$ and $\mathbf{P}_{2}$ phased at $(\phi)$ on two respective circles in any number of multiple dimensions, can equivalently be expressed as $\overrightarrow{\boldsymbol{d}}(\phi)$ (76) in terms of function vectors $\vec{f}_{1}(77)$ and $\vec{f}_{2}$ (77), which are equal to $\overrightarrow{\boldsymbol{a}}(67)-(68)$ and $\overrightarrow{\boldsymbol{b}}$ (70) - (71) vectors, respectively, and have magnitudes $f_{1}(78)$ and $f_{2}(78)$, in the direction of unit vectors $\hat{f}_{1}(77)$ and $\hat{f}_{2}(77)$, respectively. 


$$
\begin{aligned}
& \overrightarrow{\boldsymbol{d}}(\phi)= \vec{f}_{1} \operatorname{Cos} \phi+\vec{f}_{2} \operatorname{Sin} \phi+\vec{\ell}(\phi) \quad \text { where } \quad \overrightarrow{\boldsymbol{r}}_{1}(\phi)-\overrightarrow{\boldsymbol{r}}_{2}(\phi)=\vec{f}_{1} \operatorname{Cos} \phi+\vec{f}_{2} \operatorname{Sin} \phi \\
& \vec{f}_{1}=\hat{f}_{1} f_{1}=\overrightarrow{\boldsymbol{a}} \quad[(67)-(68)] \quad ; \quad \vec{f}_{2}=\hat{f}_{2} f_{2}=\overrightarrow{\boldsymbol{b}} \quad[(70)-(71)] \\
& \vec{f}_{1} \cdot \vec{f}_{1}=f_{1}^{2} \quad ; \quad\left|\vec{f}_{1}\right|=f_{1}=\sqrt{\vec{f}_{1} \cdot \vec{f}_{1}} ; \quad \vec{f}_{2} \cdot \vec{f}_{2}=f_{2}^{2} \quad ; \quad\left|\vec{f}_{2}\right|=f_{2}=\sqrt{\vec{f}_{2} \cdot \vec{f}_{2}}
\end{aligned}
$$

The vector distance $\overrightarrow{\boldsymbol{d}}(\phi)$ (76) can also be expressed as $\overrightarrow{\boldsymbol{d}}(\phi)$ (79), in terms of virtual vectors $\overrightarrow{\mathbf{X}}(\phi)(80)$ and $\overrightarrow{\mathbf{Y}}(\phi)(81)$, defined utilizing $\vec{\ell}(\phi)(62)$ and $\vec{f}_{2}(77)$.

$$
\begin{gathered}
\overrightarrow{\boldsymbol{d}}(\phi)=\vec{f}_{1} \operatorname{Cos} \phi+\vec{f}_{2} \operatorname{Sin} \phi+\vec{\ell}(\phi)=\operatorname{Cos} \phi\left[\vec{f}_{1}+\vec{f}_{2} \frac{\operatorname{Sin} \phi}{\operatorname{Cos} \phi}+\frac{\vec{\ell}(\phi)}{\operatorname{Cos} \phi}\right]=\operatorname{Cos} \phi\left[\vec{f}_{1}+\overrightarrow{\mathbf{X}}(\phi)+\overrightarrow{\mathbf{Y}}(\phi)\right] \\
\overrightarrow{\mathbf{X}}(\phi)=\frac{\vec{\ell}(\phi)}{\operatorname{Cos} \phi} ; \overrightarrow{\mathbf{X}}(\phi) \cdot \overrightarrow{\mathbf{X}}(\phi)=X^{2}(\phi)=\frac{\vec{\ell}(\phi) \cdot \vec{\ell}(\phi)}{\operatorname{Cos}^{2} \phi}=\frac{\ell^{2}(\phi)}{\operatorname{Cos}^{2} \phi} ;|\overrightarrow{\mathbf{X}}(\phi)|=X(\phi) \\
\overrightarrow{\mathbf{Y}}(\phi)=\vec{f}_{2} \frac{\operatorname{Sin} \phi}{\operatorname{Cos} \phi} ; \overrightarrow{\mathbf{Y}}(\phi) \cdot \overrightarrow{\mathbf{Y}}(\phi)=Y^{2}(\phi)=\vec{f}_{2} \cdot \vec{f}_{2} \frac{\operatorname{Sin}^{2} \phi}{\operatorname{Cos}^{2} \phi}=f_{2}^{2} \frac{\operatorname{Sin}^{2} \phi}{\operatorname{Cos}^{2} \phi} ;|\overrightarrow{\mathbf{Y}}(\phi)|=Y(\phi) \\
\operatorname{Cos} \phi \simeq k=\text { constant } \quad(\text { miniscule part of a cycle of } \phi)
\end{gathered}
$$

Within a miniscule part of a cycle of $\phi$, under special circumstances when the variation in $(\operatorname{Cos} \phi)$ is negligible compared to other parameters involved such that $(\operatorname{Cos} \phi \simeq k)$ (82) can approximately be taken as constant in this limited part of a cycle, $\overrightarrow{\boldsymbol{d}}(\phi)$ (79) would behave like the distance of points on a hyperbola ${ }^{10}(83)$ - (85), whose locations are determined by the $[\overrightarrow{\mathbf{X}}(\phi)+\overrightarrow{\mathbf{Y}}(\phi)](80)-(81)$ sum vector, from a point whose location is determined by the vector $\left[-\vec{f}_{1}\right](77)$, multiplied by a near-constant coefficient $k(82)$. The semi-major ${ }^{10}$ axis of this hyperbola ${ }^{10}(83)$ - (85) would be $\ell(\phi)(63)$ and its semi-minor ${ }^{10}$ axis would be $f_{2}(78)$.

$$
\begin{aligned}
& \frac{\overrightarrow{\mathbf{X}}(\phi) \cdot \overrightarrow{\mathbf{X}}(\phi)}{\vec{\ell}(\phi) \cdot \vec{\ell}(\phi)}-\frac{\overrightarrow{\mathbf{Y}}(\phi) \cdot \overrightarrow{\mathbf{Y}}(\phi)}{\vec{f}_{2} \cdot \vec{f}_{2}}=\frac{X^{2}(\phi)}{\ell^{2}(\phi)}-\frac{Y^{2}(\phi)}{f_{2}^{2}}=\frac{1}{\operatorname{Cos}^{2} \phi}-\frac{\operatorname{Sin}^{2} \phi}{\operatorname{Cos}^{2} \phi}=1 \\
\Rightarrow & \frac{\overrightarrow{\mathbf{X}}(\phi) \cdot \mathbf{\mathbf { X }}(\phi)}{\vec{\ell}(\phi) \cdot \vec{\ell}(\phi)}-\frac{\overrightarrow{\mathbf{Y}}(\phi) \cdot \overrightarrow{\mathbf{Y}}(\phi)}{\vec{f}_{2} \cdot \vec{f}_{2}}=1 \quad\left(\begin{array}{l}
\text { Definition of Vector Hyperbola } \\
\text { in Multi-Dimensions }
\end{array}\right) \\
\Rightarrow & \frac{X^{2}(\phi)}{\ell^{2}(\phi)}-\frac{Y^{2}(\phi)}{f_{2}^{2}}=1 \quad \text { (Definition of Scalar Hyperbola in 2-Dimensions) }
\end{aligned}
$$


Hence, due to the validity of the relation in (83), we have defined a "vector hyperbola" (84) for the vector pair $[\overrightarrow{\mathbf{X}}(\phi), \overrightarrow{\mathbf{Y}}(\phi)](80)$ - (81) based on a "scalar hyperbola"10، (85) equation for its magnitude pair $[X(\phi), Y(\phi)](80)$ - (81), respectively.

The near-hyperbolic trajectories of some comets ${ }^{11}$ when they approach the Sun could possibly be explained with this $\overrightarrow{\boldsymbol{d}}(\phi)$ (79) pattern obeying (83) - (85), considering that the comet might in fact be moving around an individual circular orbit with radius and period which is extremely large compared to that of the Sun, such that the duration it spends around the Sun is a miniscule part (82) of the total individual cycle of the comet, based on the new Circular Orbital Model for moving bodies in the Universe asserted in the book "Everyhing Is A Circle: A New Model For Orbits Of Bodies In The Universe."

Another special case is when the $\vec{\ell}(\phi)$ (62) vector, which is between the centers of these two circles in multiple dimensions, is a function of $\left(\operatorname{Cos}^{2} \phi\right)$ and cancelling out the $\left(\vec{f}_{2} \operatorname{Sin} \phi\right)$ term in the distance vector $\overrightarrow{\boldsymbol{d}}(\phi)(76)$ as in $\vec{\ell}(\phi)$ (86) [or equivalently a function of $\left(\operatorname{Sin}^{2} \phi\right)$ and cancelling out the $\left(\vec{f}_{1} \operatorname{Cos} \phi\right)$ term in the distance vector $\overrightarrow{\boldsymbol{d}}(\phi)$ (76)], in which case the distance vector $\overrightarrow{\boldsymbol{d}}(\phi)(76)$ takes the form of $\overrightarrow{\boldsymbol{d}}(\phi)(87)$.

$$
\begin{gathered}
\vec{\ell}=\vec{\ell}(\phi)=\frac{\hat{f}_{1}}{2}\left(f_{1}^{2} \operatorname{Cos}^{2} \phi+1\right)-\vec{f}_{2} \operatorname{Sin} \phi \\
\overrightarrow{\boldsymbol{d}}(\phi)=\vec{f}_{1} \operatorname{Cos} \phi+\vec{f}_{2} \operatorname{Sin} \phi+\vec{\ell}(\phi) \\
=\frac{\hat{f}_{1}}{2} f_{1}^{2} \operatorname{Cos}^{2} \phi+\vec{f}_{1} \operatorname{Cos} \phi+\frac{\hat{f}_{1}}{2}=\frac{\hat{f}_{1}}{2}\left(f_{1} \operatorname{Cos} \phi+1\right)^{2}=\frac{\hat{f_{1}}}{2}[X(\phi)+1]^{2}=\hat{f}_{1} Y(\phi)=\overrightarrow{\mathbf{Y}}(\phi) \\
\left.\left\{\begin{array}{l}
\overrightarrow{\mathbf{X}}(\phi)=\hat{f}_{1} X(\phi)=\hat{f}_{1} f_{1} \operatorname{Cos} \phi=\vec{f}_{1} \operatorname{Cos} \phi \\
\overrightarrow{\mathbf{Y}}(\phi)=\hat{f}_{1} Y(\phi)=\frac{\hat{f}_{1}}{2}\left(f_{1} \operatorname{Cos} \phi+1\right)^{2}=\frac{\hat{f}_{1}}{2}[X(\phi)+1]^{2}
\end{array}\right\} \begin{array}{l}
\text { Definition of Vector Parabola } \\
\text { coordinates in Multi-Dim.sions }: \\
\text { Focus distance } f \text { \&ertex }[1,0]
\end{array}\right) \\
Y(\phi)=\frac{1}{2}[X(\phi)+1]^{2}=\frac{1}{4 f}[X(\phi)+1]^{2}=\frac{1}{4 f} X^{2}(\phi)+\frac{1}{2 f} X(\phi)+\frac{1}{4 f} \Rightarrow f=\frac{1}{2}
\end{gathered}
$$

(Definition of Scalar Parabola in 2-Dimensions with focus distance $f$ \& vertex $[1,0]$ )

As a result, in this special case, the distance vector $\overrightarrow{\boldsymbol{d}}(\phi)(87)$ at any value of the phase $\phi$, between points $\mathbf{P}_{1}$ phased at $\left(\phi+\phi_{0}\right)$ and $\mathbf{P}_{2}$ phased at $(\phi)$ on two respective circles in any 
number of multiple dimensions, would behave like the $\overrightarrow{\mathbf{Y}}(\phi)$-coordinate (88) of points on a "vector parabola" ${ }^{12 "}$ (88) formed by the vector pair $[\overrightarrow{\mathbf{X}}(\phi), \overrightarrow{\mathbf{Y}}(\phi)](88)$, with a focus ${ }^{12}$ distance of $\left(f=\frac{1}{2}\right)(89)$ and vertex ${ }^{12}[1,0]$, based on the scalar parabola ${ }^{12}$ equation (89) that the magnitude pair $[X(\phi), Y(\phi)](88)$ - (89) obeys.

Considering the new Circular Orbital Model for moving bodies in the Universe asserted in the book "Everyhing Is A Circle: A New Model For Orbits Of Bodies In The Universe", the parabolic ${ }^{13}$ escape trajectories that moving objects follow while moving away from Earth or other bodies asserting a gravitational pull could possibly be explained with this $\vec{d}(\phi)(87)$ pattern obeying (88) - (89), as these objects are originally moving together around the same individual circular orbit of the pulling body, and the parabolic ${ }^{13}$ escape trajectory is based on a shift of $\vec{\ell}(\phi)$ (86) vector between their centers of revolution, with some cancellation of the dependance of escaping object from original trajectory of the pulling body.

\section{CONCLUSIONS}

The main result presented in this Article is that the vector distance between two points on two respective circles in multiple number of dimensions can equivalently be mathematically expressed and interpreted as the distance of points on a virtual vector ellipse from another fixed or moving point. This result is mathematically valid even when the phase difference of the two points on their respective circles is a variable function of time. The conditions for the special cases of parabola and hyperbola are also investigated and demonstrated. Alongside this mathematical derivation and proof, concepts of "vector ellipse", "vector hyperbola", and "vector parabola" are also introduced and mathematically defined.

\section{AUTHOR CONTRIBUTIONS}

Aslı Pinar Tan is the only author contributing to this Article.

\section{DATA AVAILIBILITY}

Data sharing is not applicable to this article as no data were created or analyzed in this study. 


\title{
DECLARATION OF INTEREST STATEMENT
}

The Author declares that there is no conflict of interest.

\begin{abstract}
KEYWORDS
Conic Sections, Topology, Circle, Ellipse, Hyperbola, Parabola, Orbit, Trajectory, Orbital Mechanics, Solar System, Planetary System, Planet, Satellite, Comet, Sun, Earth, Moon
\end{abstract}

\begin{abstract}
ABOUT THE AUTHOR
Aslı P1nar Tan's findings in this Article are part of all her findings as a result of her personal theoretical studies and research over the years independent from any institution or university, which she has published in the book "Everyhing Is A Circle: A New Model For Orbits Of Bodies In The Universe"4, and will further publish in a set of books and other articles. She is an Electrical \& Electronics Engineer, Bsc. 1995 and Msc. 1997 graduated from Bilkent University, Ankara, Turkey, and she also holds a degree in Multi-Disciplinary Space Studies program graduated in 2000 from International Space University, Strasbourg, France. She has worked professionally more than 20 years in the Global ICT / Telecom industry, in addition to her academic career in Electromagnetics and Space Applications, all independent from this research. She has been granted a Young Scientist Award by URSI (Union Radio Science Internationale) in 1998 based on her Master Thesis Research results presenting a systematic methodology for High-Frequency Radar Antenna Design using the Genetic Algorithm and Equivalent Edge Currents, to generate plain waves in the near field, which is utilized in the industry since then. (For more: http://www.linkedin.com/in/apinartan)
\end{abstract}




\section{REFERENCES}

References in this Article can be any Physics and Calculus textbook, as the physics observations and mathematical equations and identities used as a basis for the proof are all currently accepted theory in existing textbooks.

1. “Dot product”, Wikipedia, https://en.wikipedia.org/wiki/Dot_product (2021)

2. "Ellipse”, Wikipedia, http://en.wikipedia.org/wiki/Ellipse (2021)

3. "Semi-major and semi-minor axes", Wikipedia, http://en.wikipedia.org/wiki/Semi-major_and_semi-minor_axes (2021)

4. A.P. Tan, "Everything is a Circle: A New Model For Orbits of Bodies in the Universe", Amazon Kindle Publishing ISBN \& EAN 979-8-56-757466-9 (2020) Paperback Book: https:/www.amazon.com/gp/product/B08NYG14X8 Kindle eBook: https://www.amazon.com/gp/product/B08PVS2FBW

5. "Focus (geometry)", Wikipedia, http://en.wikipedia.org/wiki/Focus_(geometry) (2021)

6. “Sun”, Wikipedia, https://en.wikipedia.org/wiki/Sun (2021)

7. “Earth”, Wikipedia, https://en.wikipedia.org/wiki/Earth (2021)

8. “Earth's orbit”, Wikipedia, https://en.wikipedia.org/wiki/Earth\%27s_orbit (2021)

9. “Moon”, Wikipedia, https://en.wikipedia.org/wiki/Moon (2021)

10. "Hyperbola", Wikipedia, http://en.wikipedia.org/wiki/Hyperbola (2021)

11. "List of hyperbolic comets", Wikipedia, http://en.wikipedia.org/wiki/List_of_hyperbolic_comets (2021)

12. "Parabola", Wikipedia, http://en.wikipedia.org/wiki/Parabola (2021)

13. "Parabolic trajectory", Wikipedia, http://en.wikipedia.org/wiki/Parabolic trajectory (2021) 